\title{
GROUPOIDS IN SIKORSKI'S SPACES, CONNECTIONS AND THE CHERN-WEIL HOMOMORPHISM
}

\author{
KRZYSZTOF LISIECKI \\ Institute of Mathematics, Technical University of Eódź \\ al. Politechniki 11, 90-924 Eódź, Poland \\ E-mail: lisiecki@ck-sg.p.lodz.pl
}

\begin{abstract}
The subject of this paper is the notion of the connection in a foliated groupoid (briefly, F-groupoid) and the construction of the Chern-Weil homomorphism of an F-groupoid. The author shows its independence of the choice of the connection.

1. Introduction. Relations between differential groupoids, their algebroids and principal bundles have pushed many mathematicians to work over such notions as connection or Chern-Weil homomorphism. There appeared many new sources of Lie groupoids and Lie algebroids. They are, first of all, vector bundles, Poisson manifolds, transversally complete foliations. At the same time, foliations are a rich source of non-transitive groupoids, in which the space is not a manifold, in general. Examples of such groupoids are the so-called Pradines-type groupoids. The author of this paper inspired by [K1] defined the notion of a foliated groupoid (briefly, F-groupoid) over a foliation with singularities in the sense of P. Stefan [ST1], [ST2] (see also [K4]).

Examples of F-groupoids are Pradines-type groupoids [K2] and the groupoid constructed as follows: for any fixed Lie groupoid $(\Psi, \alpha, \beta, M, \cdot)$ and any foliation with singularities in the sense of Stefan $E \subset T M$, we put $\Phi:=(\alpha, \beta)^{-1}[R]$ where $R$ is an equivalence relation determined by the foliation $E$.

Let $(A, \llbracket \cdot, \cdot \rrbracket, \gamma)$ be a Lie algebroid on a differential manifold $M$.

If $\gamma$ is of a constant rank, then $\operatorname{Im} \gamma$ is a foliation without singularities and the Lie algebroid is called regular. In the opposite case, $\operatorname{Im} \gamma$ is a foliation with singularities in the sense of Stefan and the algebroid is called nonregular. In the present paper the author builds the theory of connections in such algebroids.
\end{abstract}

2000 Mathematics Subject Classification: Primary 58H05.

Key words and phrases: smooth groupoid, foliated groupoid, foliated algebroid, connection, Chern-Weil homomorphism.

The paper is in final form and no version of it will be published elsewhere. 
For a regular Lie algebroid $A$ over the manifold $M$, by a connection we mean a splitting $\lambda$ of the short exact Atiyah sequence $0 \rightarrow k e r \gamma \hookrightarrow A \stackrel{\gamma}{\rightarrow} \operatorname{Im} \gamma \rightarrow 0$. For any transitive Lie algebroid $A$ over the manifold $M$ for which the short exact Atiyah sequence is $0 \rightarrow \operatorname{ker} \gamma \hookrightarrow A \stackrel{\gamma}{\rightarrow} T M \rightarrow 0$ and for any Stefan's foliation $E \subset T M$, the inverse image $A^{\prime}=\gamma^{-1}(E)$ of the foliation $E$ by the mapping $\gamma$ is a nonregular algebroid. $A^{\prime}$ is not a vector bundle! But this algebroid possesses a connection which is a restriction of any connection in $A$. Moreover, if the foliation $E$ does not have a constant rank and $A$ is a vector bundle, then a splitting of the short exact Atiyah sequence does not exist. So the connections in the algebroids over Stefan's foliations appear if the algebroid is built on a more general structure than a vector bundle. It appears that the most useful is the notion of a vector semibundle which admits the existence of fibres of different dimensions, but we have to go beyond the category of differential manifolds, into the category of differential spaces. The basic difficulty in the building of connections in an algebroid over the Stefan foliation is that there does not exist a local base of the module of cross-sections of a semibundle. We can avoid these difficulties if the algebroid comes from an F-groupoid. The second part of this paper contains a construction of the Chern-Weil homomorphism of the F-groupoid and the proof of its independence of the choice of the connection in the F-groupoid.

In 1999 J. Huebschmann $[\mathrm{H}]$ has considered Lie-Rinehart algebras and their extensions. F-algebroids determine examples of Lie-Rinehart algebras. J. Huebschmann has constructed the Chern-Weil homomorphism for extensions of Lie-Rinehart algebras. The precise comparison of the constructions of Chern-Weil homomorphisms will be done in another paper of the author.

2. Differential spaces. Let $\mathfrak{C}$ be any non-empty family of real functions defined on the set $M$, and $\tau_{M}$ the weakest topology under which all functions from $\mathfrak{C}$ are continuous. Let $A \subset M$. We put

$$
\mathfrak{C}_{A}=\left\{\beta: A \rightarrow \mathbb{R} ; \bigwedge_{x \in A} \bigvee_{x \in U \in \tau_{\mathfrak{C}}} \bigvee_{\alpha \in \mathfrak{C}}(\beta|A \cap U=\alpha| A \cap U)\right\}
$$

and

$$
s c \mathfrak{C}=\left\{\phi \circ\left(\alpha_{1}(\cdot), \ldots, \alpha_{m}(\cdot)\right) ; \alpha_{1}, \ldots, \alpha_{m} \in \mathfrak{C}, \phi \in C^{\infty}\left(\mathbb{R}^{m}\right) ; m \in \mathbb{N}\right\} .
$$

The family $\mathfrak{C}$ is called a differential structure on $M$ if $\mathfrak{C}_{M}=\mathfrak{C}$ and $\operatorname{sc} \mathfrak{C}=C$. The pair $(M, \mathfrak{C})$ is called a (Sikorski's) differential space [SI].

A differential space $(N, \mathfrak{D})$ will be called a differential subspace of a differential space $(M, \mathfrak{C})[\mathrm{K} 2]$, if $N \subset M$ and, if for each point $y \in N$ there is a neighbourhood $U \in \tau_{\mathfrak{D}}$ of the point $y$ such that $\mathfrak{D}_{U}=\mathfrak{C}_{U}$. If $\mathfrak{D}=\mathfrak{C}_{N}$, then $(N, \mathfrak{D})$ will be called a proper differential subspace of a differential space $(M, \mathfrak{C})$.

Let $(M, \mathfrak{C})$ and $(N, \mathfrak{D})$ be any differential spaces. The mapping $f: M \rightarrow N$ is called smooth if $g \circ f \in \mathfrak{C}$ for $g \in \mathfrak{D}$.

\section{The category of vector semibundles}

Definition 3.1. By a vector semibundle over a differential space $M$ we mean a system $\mathfrak{f}=\left(\mathfrak{f}, p, M,\left\{\mathfrak{f}_{1 x}\right\}_{x \in M}\right)$, in which $\mathfrak{f}$ is a differential space, $p: \mathfrak{f} \rightarrow M$ is a smooth 
mapping, $\mathfrak{f}_{1 x}=p^{-1}(x)$ is a set equipped with the structure of a real vector space and the following axioms are fulfilled:

1. the mappings $+: \mathfrak{f} * \mathfrak{f} \rightarrow \mathfrak{f}$, where $\mathfrak{f} * \mathfrak{f}=\{(u, v) \in \mathfrak{f} \times \mathfrak{f}: p(u)=p(v)\}$ and . : $\mathbb{R} \times \mathfrak{f} \rightarrow \mathfrak{f}$ are defined as the ordinary addition of vectors in the same vector space $\mathfrak{f}_{1 x}$ and the multiplication of vectors by a number, are smooth,

2. the differential structure $\mathfrak{C}(f)$ of the differential space $\mathfrak{f}$ is identical with the structure $\left(\operatorname{sc}\left(\mathfrak{C}_{0}(f)\right)\right)_{\mathfrak{f}}$ where

$$
\mathfrak{C}_{0}(f)=\{\delta \circ p ; \delta \in C(M)\} \cup\left\{\delta \in \mathfrak{C}(f) ; \bigwedge_{x \in M} \delta \mid \mathfrak{f}_{x} \text { is a linear mapping }\right\} .
$$

Definition 3.2. Let $\left(\mathfrak{f}^{1}, p^{1}, M,\left\{\mathfrak{f}_{1 x}^{1}\right\}_{x \in M}\right)$ and $\left(\mathfrak{f}^{2}, p^{2}, M,\left\{\mathfrak{f}_{\mid x}^{2}\right\}_{x \in M}\right)$ be two semibundles over the same differential space $M$. By a (strong) homomorphism of semibundles $\mathfrak{f}^{1}$ and $\mathfrak{f}^{2}$ we mean a smooth mapping $S: \mathfrak{f}^{1} \rightarrow \mathfrak{f}^{2}$ such that

(1) $p^{2} \circ S=p^{1}$

(2) for each $x \in M$ the mapping $S_{1 x}: \mathfrak{f}_{\mid x}^{1} \rightarrow \mathfrak{f}_{1 x}^{2}$ is linear.

Definition 3.3. By a vector subsemibundle of the vector semibundle $\mathfrak{f}=(\mathfrak{f}, p, M$, $\left.\left\{\mathfrak{f}_{1 x}\right\}_{x \in M}\right)$ we mean a vector semibundle $\mathfrak{g}=\left(\mathfrak{g}, p, M,\left\{\mathfrak{g}_{1 x}\right\}_{x \in M}\right)$ such that $\mathfrak{g}$ is a proper differential subspace of the differential space $\mathfrak{f}$ and the inclusion $i: \mathfrak{g} \hookrightarrow \mathfrak{f}$ is a smooth homomorphism of vector semibundles.

Lemma 3.4. Let $\mathfrak{f}=\left(\mathfrak{f}, p, M,\left\{\mathfrak{f}_{1 x}\right\}_{x \in M}\right)$ be a vector semibundle. If $\mathfrak{g}$ is a proper differential subspace of $\mathfrak{f}$ and, for each $x \in M$, the set $\mathfrak{g} \cap p^{-1}(x) \subset \mathfrak{f}_{1 x}$ is a vector subspace of $\mathfrak{f}_{\mid x}$, then the system $\mathfrak{g}=\left(\mathfrak{g}, p \mid \mathfrak{g}, M,\left\{\mathfrak{g}_{1 x}\right\}_{x \in M}\right)$ is a vector semibundle over the differential space $M$.

All vector semibundles, together with homomorphisms, form a category. If $(M, \mathfrak{C}(M))$ is a differential space, then the differential space $(T M, \mathfrak{C}(T M))$, together with the natural projection on $M$ forms a vector semibundle. In particular, each vector bundle $\mathfrak{f}$ over the differential manifold $M$ is a vector semibundle.

Definition 3.5. By a Cartesian product of vector semibundles $\mathfrak{f}^{1}=\left(\mathfrak{f}^{1}, p^{1}, M^{1}\right.$, $\left.\left\{\mathfrak{f}_{1 x^{1}}^{1}\right\}_{x^{1} \in M^{1}}\right)$ and $\mathfrak{f}^{2}=\left(\mathfrak{f}^{2}, p^{2}, M^{2},\left\{\mathfrak{f}_{\mid x^{2}}^{2}\right\}_{x^{2} \in M^{2}}\right)$ we mean a vector semibundle

$$
\mathfrak{f}^{1} \times \mathfrak{f}^{2}=\left(\mathfrak{f}^{1} \times \mathfrak{f}^{2}, p^{1} \times p^{2}, M^{1} \times M^{2},\left\{\left(\mathfrak{f}^{1} \times \mathfrak{f}^{2}\right)_{\left(x^{1}, x^{2}\right)}\right\}_{\left.\left(x^{1}, x^{2}\right) \in M^{1} \times M^{2}\right),}\right.
$$

in which $\mathfrak{f}^{1} \times \mathfrak{f}^{2}$ is a Cartesian product of differential spaces with its natural differential structure.

Definition 3.6. Let $\mathfrak{g}=\left(\mathfrak{g}, p, M,\left\{\mathfrak{g}_{1 x}\right\}_{x \in M}\right)$ be a vector semibundle over the differential space $M$ and let $f: N \rightarrow M$ be a smooth mapping. By the pull-back of the vector semibundle $\mathfrak{g}$ by the mapping $f$ we mean a vector semibundle $f^{*} \mathfrak{g}=\left(f^{*} \mathfrak{g}, p r_{1}, N\right.$, $\left.\left\{x, \mathfrak{g}_{1 f(x)}\right\}_{x \in N}\right)$ where

$$
f^{*} \mathfrak{g}=\{(x, v) \in N \times \mathfrak{g} ; f(x)=p(v)\} \subset N \times \mathfrak{g} .
$$




\section{The category of groupoids in the category of differential spaces}

DEFINITION 4.1. By a groupoid in the category of differential spaces over a differential manifold $M$ we mean a groupoid $\Phi=(\Phi, \alpha, \beta, M, \cdot)$, in which $\Phi$ is a differential space and the mappings $\alpha, \beta,{ }^{-1}, u, \cdot$ are smooth in the category of differential spaces.

Proposition 4.2. The Cartesian product of groupoids in the category of differential spaces is a groupoid in the category of differential spaces.

For $x \in M$, we define $\tilde{\Phi}_{x}:=\alpha^{-1}(x), \tilde{G}_{x}:=(\alpha, \beta)^{-1}(x)=\left(\beta_{x}\right)^{-1}[\{x\}]$ and the mapping $\beta_{x}: \tilde{\Phi}_{x} \rightarrow \tilde{L}_{x}, h \mapsto \beta(h)$, where $\tilde{L}_{x}$ is the equivalence class of the relation $x \approx y \Longleftrightarrow \bigvee_{h \in \Phi}(\alpha h=x, \beta h=y)$. Of course, $\tilde{L}_{x}$ contains $x$. The subsets $\tilde{\Phi}_{x}$ and $\tilde{G}_{x}$ are equipped with the structures of proper differential subspaces of the differential space $\Phi$. For $x \in M$, the set $\tilde{G}_{x}$, together with the action induced from the groupoid $\Phi$, forms a group. Moreover, the multiplication and taking inverse of elements in $\tilde{G}_{x}$ are smooth (in the sense of differential spaces).

$\tilde{G}_{x}$, together with the structure of the proper differential subspace of the differential space $\Phi$, forms a generalized Lie group $[\mathrm{SA}]$.

Note that the mapping $\cdot: \tilde{\Phi}_{x} \times \tilde{G}_{x} \rightarrow \tilde{\Phi}_{x}$ is a smooth right action of the generalized Lie group $\tilde{G}_{x}$ on the differential space $\tilde{\Phi}_{x}$, orbits of which are identical with fibres of smooth mappings $\beta_{x}: \tilde{\Phi}_{x} \rightarrow \tilde{L}_{x}$. Additionally, the mapping $D_{h}: \tilde{\Phi}_{\beta h} \rightarrow \tilde{\Phi}_{\alpha h}, g \mapsto g \cdot h$, is a diffeomorphism of differential spaces. The space $\mathfrak{g}_{1 x}:=T_{u_{x}}\left(\tilde{G}_{x}\right)$ tangent to the generalized Lie group $\tilde{G}_{x}$ is equipped with a natural Lie algebra structure [SA]. Put $\mathfrak{g}:=\bigcup_{x \in M} \mathfrak{g}_{1 x} \subset$ $T \Phi$. The differential structure on the set $\mathfrak{g}$ is induced from the differential space $T \Phi$.

Lemma 4.3. The system $\left(\mathfrak{g}, p, M,\left\{\mathfrak{g}_{1 x}\right\}_{x \in M}\right)$, in which $p: \mathfrak{g} \rightarrow M$ is the projection defined by $p^{-1}(x)=\mathfrak{g}_{1 x}$, is a vector semibundle over the differential manifold $M$.

$\tilde{T}^{\alpha} \Phi=\bigcup_{h \in \Phi} T_{h} \tilde{\Phi}_{\alpha h} \subset T \Phi$ is a vector subsemibundle.

The module $\mathfrak{X}^{R}(\Phi)$ of smooth right invariant vector fields on $\Phi$ forms a module over the ring $C^{\infty}(M)$ with respect to the addition of vector fields and their multiplication by functions $f \bullet X=f \circ \beta \cdot X, f \in C^{\infty}(M)$.

The set of all smooth $\alpha$-fields on $\Phi$, i.e. the set of all smooth cross-sections of the semibundle $\tilde{T}^{\alpha} \Phi$, is denoted by $\mathfrak{X}^{\alpha}(\Phi)$.

Definition 4.4. Let $\Phi$ and $\mathfrak{f}$ be a groupoid in the category of differential spaces and a vector semibundle, both over the same differential manifold $M$. By a representation of the groupoid $\Phi$ in the semibundle $\mathfrak{f}$ we mean the smooth mapping $T: \Phi * \mathfrak{f} \rightarrow \mathfrak{f}$, ( $\Phi * \mathfrak{f}=\{(h, v) \in \Phi \times \mathfrak{f} ; \alpha(h)=p(v)\} \subset \Phi \times \mathfrak{f}$ is a proper differential subspace of $\Phi \times \mathfrak{f})$, which fulfils the following conditions:

1. $p \circ T(h, v)=\beta(h)$, for $(h, v) \in \Phi * \mathfrak{f}$,

2. for any $h \in \Phi$, the mapping $T(h): \mathfrak{f}_{\mid \alpha h} \rightarrow \mathfrak{f}_{1 \beta h}, v \mapsto T(h, v)$, is a linear isomorphism,

3. $T(g \cdot h)=T(g) \circ T(h)$ for $(g, h) \in \Phi * \Phi$,

4. $T\left(u_{x}\right)=i d_{\mathfrak{f}_{1 x}}, x \in M$.

The trivial representation of $\Phi$ in $\mathfrak{f}=M \times \mathbb{R}$ is defined by $T(h)=i d_{\mathbb{R}}$. 
DEFINITION 4.5. By the adjoint representation of the groupoid $\Phi$ we mean the mapping $\widetilde{A d}: \Phi * \mathfrak{g} \rightarrow \mathfrak{g}$ which is defined by the formula $\widetilde{A d}(h)=\left(\tilde{\tau}_{h}\right)_{* u_{\alpha h}}: \mathfrak{g}_{1 x} \rightarrow \mathfrak{g}_{1 y}$ where $\tilde{\tau}_{h}: \tilde{G}_{x} \rightarrow \tilde{G}_{y}, a \mapsto h \cdot a \cdot h^{-1}$ for $h \in \Phi$ such that $\alpha h=x$ and $\beta h=y$.

THEOREM 4.6. $\widetilde{\mathrm{Ad}}$ is a representation of the groupoid $\Phi$ in the semibundle $\mathfrak{g}$.

Proof. We shall show the smoothness of the mapping $\widetilde{A d}: \Phi * \mathfrak{g} \rightarrow \mathfrak{g}$. The differential structure of $\mathfrak{g} \subset T \Phi$ is generated by the set $\{f \circ \pi \mid \mathfrak{g} ; f \in \mathfrak{C}(\Phi)\} \cup\{(d f) \mid \mathfrak{g} ; f \in \mathfrak{C}(\Phi)\}$. It is enough to prove that for any $f \in \mathfrak{C}(\Phi)$ we have

1. $f \circ \pi \mid \mathfrak{g} \circ \widetilde{A d} \in\left(\mathfrak{C}(\Phi) \times \mathfrak{C}(T \Phi)_{\mathfrak{g}}\right)_{\Phi * \mathfrak{g}}$,

2. $(d f) \mid \mathfrak{g} \circ \widetilde{A d} \in\left(\mathfrak{C}(\Phi) \times \mathfrak{C}(T \Phi)_{\mathfrak{g}}\right)_{\Phi * \mathfrak{g}}$.

(1) Let $f \in \mathfrak{C}(\Phi)$ and $(h, v) \in \Phi * \mathfrak{g}$. Then: $f \circ \pi \mid \mathfrak{g} \circ \widetilde{A d}(h, v)=f \circ \pi(\widetilde{A d}(h)(v))=$ $f\left(u_{\beta h}\right)$, because $\widetilde{A d}(h)(v) \in T_{u_{\beta h}} \Phi_{\beta h}$, so $\pi(\widetilde{A d}(h)(v))=u_{\beta h}$. So we get $(f \circ \pi \mid \mathfrak{g}) \circ$ $\widetilde{A d}(h, v)=f \circ u \circ \beta \circ p r_{1} \mid \Phi * \mathfrak{g} \in\left(\mathfrak{C}(\Phi) \times \mathfrak{C}(T \Phi)_{\mathfrak{g}}\right)_{\Phi * \mathfrak{g}} \cdot$

(2) $(d f)|\mathfrak{g} \circ \widetilde{A d}(h, v)=(d f)| \mathfrak{g} \circ \widetilde{A d}(h)(v)=(d f)\left(\left(\tau_{h}\right)_{* u_{\alpha h}}(v)\right)=\left(\tau_{h}\right)_{* u_{\alpha h}}(v)(f)=$ $\left.\left(\tau_{h}\right)_{* u_{\alpha h}}(v)\left(f \mid \tilde{G}_{\beta h}\right)=v\left(f \mid \tilde{G}_{\beta h} \circ \tau_{h}\right)=v\left(\tilde{G}_{\alpha h} \ni a\right) \mapsto f\left(h a h^{-1}\right)\right)=v\left(\tilde{G}_{\alpha h} \ni a\right) \mapsto$ $f \circ \rho(a,(h, v))$, where $\rho: \Phi *(\Phi * \mathfrak{g}) \rightarrow \Phi,(a,(h, v)) \mapsto h \cdot a \cdot h^{-1}$, and $\Phi *(\Phi * \mathfrak{g})=$ $\{(a,(h, v)) \in \Phi \times(\Phi \times \mathfrak{g}) ; \alpha a=\beta a=\beta h\}$. By the smoothness of the multiplication on $\Phi$, we have $f \circ \rho \in\left(\mathfrak{C}(\Phi) \times \mathfrak{C}(T \Phi)_{\mathfrak{g}}\right)_{\Phi * \mathfrak{g}}$. Put $N=\Phi * \mathfrak{g}$ and $Y: N \rightarrow T \Phi,(h, v) \mapsto v$. The mapping $\mathrm{Y}$ is smooth, of course. Fix $\left(h_{0}, v_{0}\right) \in \Phi * \mathfrak{g}$ and find an open neighbourhood $\Omega \subset \Phi \times(\Phi * \mathfrak{g})$ of the point $\left(u_{\alpha h_{0}},\left(h_{0}, v_{0}\right)\right)$ and the mapping $\tilde{f} \in\left(\mathfrak{C}(\Phi) \times \mathfrak{C}(T \Phi)_{\mathfrak{g}}\right)_{\Phi * \mathfrak{g}}$. such that

$$
f \circ \rho|\Omega *(\Phi *(\Phi * \mathfrak{g}))=\tilde{f}| \Omega *(\Phi *(\Phi * \mathfrak{g})) .
$$

Then, for the point $(h, v)$ from some neighbourhood of the point $\left(h_{0}, v_{0}\right) \in \Phi * \mathfrak{g}$ we have:

$$
\begin{aligned}
((d f) \mid \mathfrak{g}) \circ \widetilde{A d}(h, v) & =v\left(\tilde{G}_{\alpha h} \ni a \mapsto f \circ \rho(a,(h, v))\right) \\
& =v(\tilde{f}(\cdot,(h, v)))=Y(h, v)(\tilde{f}(\cdot,(h, v))) .
\end{aligned}
$$

Now, the fact that $(d f) \mid \mathfrak{g}) \circ \widetilde{A d} \in\left(\mathfrak{C}(\Phi) \times \mathfrak{C}(T \Phi)_{\mathfrak{g}}\right)_{\Phi * \mathfrak{g}}$ we get from the lemma below putting: $M=\Phi * \mathfrak{g}$ and $N=\Phi *(\Phi * \mathfrak{g})$.

LEMmA 4.7. For differential spaces $(M, \mathfrak{C}(M))$ and $(N, \mathfrak{D}(N))$, a smooth mapping $Y:(N, \mathfrak{D}(N)) \rightarrow(T M, \mathfrak{C}(T M))$ and a smooth function $g: M \times N \rightarrow \mathbb{R}$, the function $N \ni x \mapsto Y(x)(g(\cdot, x)) \in \mathbb{R}$, belongs to $\mathfrak{D}(N)$.

Notation. Let $T$ be a representation of the groupoid $\Phi$ in the vector semibundle $f$ and let $\sigma \in \operatorname{Sec} \mathfrak{f}$ be any smooth cross-section of the semibundle $\mathfrak{f}$. Denote

$$
\sigma^{T}: \Phi \rightarrow \mathfrak{f}, \quad h \mapsto T\left(h^{-1}\right)(\sigma \circ \beta(h)) .
$$

The mapping $\sigma^{T}$ is smooth and $\sigma^{T}\left[\tilde{\Phi}_{x}\right] \subset \mathfrak{f}_{1 x}$ for $x \in M$. So, $\sigma^{T}$ induces the family of mappings $\sigma_{1 x}^{T}: \tilde{\Phi}_{x} \rightarrow \mathfrak{f}_{1 x}$.

THEOREM 4.8. For any smooth $\alpha$-field $\xi \in \tilde{X}^{\alpha}(\Phi)$ on $\Phi$ and for a smooth mapping $\sigma: \Phi \rightarrow \mathfrak{f}$ such that $\sigma\left[\tilde{\Phi}_{x}\right] \subset \mathfrak{f}_{1 x}$, the mapping $\xi(\sigma): \Phi \rightarrow \mathfrak{f}, h \mapsto \xi_{h}\left(\sigma \mid \tilde{\Phi}_{\alpha h}\right)$, is smooth.

Proof. To prove the smoothness of $\xi(\sigma)$, we have to show the smoothness of the mapping $\delta \circ \xi(\sigma)$ for any function $\delta \in \mathfrak{C}(\mathfrak{f})$. The differential structure of $\mathfrak{C}(\mathfrak{f})$ is generated 
by the set $\left\{\delta_{1} \circ p ; \delta_{1} \in C^{\infty}(M)\right\} \cup\left\{\delta_{1} \in \mathfrak{C}(\mathfrak{f}) ; \delta_{1} \mid \mathfrak{f}_{1 x}\right.$ is a linear mapping $\}$, so it is enough to check the smoothness of $\delta \circ \xi(\sigma)$ for any $\delta$ from this set.

(a) Let $\delta=\delta_{1} \circ p$ for $\delta_{1} \in C^{\infty}(M)$. Then $\delta \circ \xi(\sigma)=\delta_{1} \circ p \circ \xi(\sigma)=\delta_{1} \circ \alpha$, and the function $\delta \circ \xi(\sigma)$ is smooth.

(b) Let $\delta \in \mathfrak{C}(\mathfrak{f})$ be a linear mapping in each fibre $\mathfrak{f}_{1 x}, x \in M$. We define $\delta_{x}=$ $\delta \mid \tilde{\Phi}_{x}: \tilde{\Phi}_{x} \rightarrow \mathfrak{f}_{\mid x}$. Then $\delta \circ \xi(\sigma)(h)=\delta\left(\xi_{h}\left(\sigma_{\mid \alpha h}\right)\right)=\left(\delta \mid \mathfrak{f}_{\mid \alpha h}\right)\left(\xi_{h}\left(\sigma_{\mid \alpha h}\right)\right)=$ $\xi_{h}\left(\delta \mid \mathfrak{f}_{\mid \alpha h} \circ \sigma_{\mid \alpha h}\right)=\xi(\delta \circ \sigma)(h)$. And so, $\delta \circ \xi(\sigma)=\xi(\delta \circ \sigma)$, but $\delta \circ \sigma: \Phi \rightarrow \mathbb{R}$ is smooth as a superposition of smooth mappings. Then $\xi(\delta \circ \sigma)$ is a smooth function on $\Phi$.

Corollary 4.9. The mapping $\xi(\sigma) \circ u: M \rightarrow \mathfrak{f}$ is a smooth cross-section of the vector semibundle $\mathfrak{f}$.

DEFINITION 4.10. By a covariant differential operator (briefly CDO) in the vector semibundle $\mathfrak{f}$ we mean an $\mathbb{R}$-linear mapping $\mathfrak{L}: S e c \mathfrak{f} \rightarrow S e c \mathfrak{f}$ for which there exists a vector field $X \in X(M)$ such that $\mathfrak{L}(f \cdot \sigma)=X(f) \sigma+f \cdot \mathfrak{L}(\sigma)$ for $f \in C^{\infty}(M)$ and $\sigma \in S e c f$.

The vector field $X$ is called an anchor of the CDO $\mathfrak{L}$ and denoted by $q(\mathfrak{L})$. It is easy to see that the field $X$ is uniquely determined.

Theorem 4.11. For $\xi \in \mathfrak{X}^{R}(\Phi)$, the mapping $\tilde{T}^{\prime}(\xi):$ Sec $\mathfrak{f} \rightarrow \operatorname{Sec} \mathfrak{f}, \sigma \mapsto \xi\left(\sigma^{T}\right) \circ u$ is a $C D O$ with the anchor $\gamma(\xi)$.

Proof. Since for $f \in C^{\infty}(M)$ and $\sigma \in \operatorname{Sec} \mathfrak{f}$ we have $(f \cdot \sigma)^{T}=f \circ \beta \cdot \sigma^{T}$, it follows that for $\xi \in \mathfrak{X}^{R}(\Phi)$ we get

$$
\begin{aligned}
\tilde{T}^{\prime}(\xi)(f \cdot \sigma) & =\xi(f \cdot \sigma)^{T} \circ u=\left(x \mapsto \xi_{u_{x}}\left(f \circ \beta \cdot \sigma^{T}\right)\right) \\
& =\left(x \mapsto \xi_{u_{x}}(f \circ \beta) \cdot \sigma_{u_{x}}^{T}+\beta\left(u_{x}\right) \cdot \xi_{u_{x}}\left(\sigma^{T}\right)\right) \\
& =\left(x \mapsto \gamma(\xi)_{x}(f) \cdot \sigma_{x}+f(x) \cdot \tilde{T}^{\prime}(\xi)(\sigma)_{x}\right) \\
& =\gamma(\xi)(f) \cdot \sigma+f \cdot \tilde{T}^{\prime}(\xi)(\sigma) .
\end{aligned}
$$

The mapping $\tilde{T}^{\prime}(\xi)$ is $\mathbb{R}$-linear, thus a CDO in the semibundle $\mathfrak{f}$ with anchor $\gamma(\xi)$.

LEMMA 4.12. The set $C D O(\mathfrak{f})$ of all covariant differential operators in the vector semibundle $\mathfrak{f}$ forms a real Lie algebra with a bracket defined by the formula $\left[\mathfrak{L}_{1}, \mathfrak{L}_{2}\right]=$ $\mathfrak{L}_{1} \circ \mathfrak{L}_{2}-\mathfrak{L}_{2} \circ \mathfrak{L}_{1}$.

Definition 4.13. By the derivative of the representation $T: \Phi * \mathfrak{f} \rightarrow f$ of the groupoid $\Phi$ in the vector semibundle $\mathfrak{f}$ we mean the mapping $\tilde{T}^{\prime}: \mathfrak{X}^{R}(\Phi) \rightarrow C D O(\mathfrak{f}), \xi \mapsto \tilde{T}^{\prime}(\xi)$.

The mapping $\tilde{T}^{\prime}$ is a $C^{\infty}(M)$-linear homomorphism. Moreover, $\tilde{T}^{\prime}$ is a homomorphism of Lie algebras.

Let $\mathfrak{f}=M \times \mathbb{R}$ be a trivial bundle. Then, for any vector field $X \in \mathfrak{X}(M)$, the mapping $\tilde{X}: C^{\infty}(M) \rightarrow C^{\infty}(M), f \mapsto X(f)$, is a CDO.

\section{Algebroids of F-groupoids}

Definition 5.1. The distribution $E \subset T M$ on the differential space $(M, \mathfrak{C}(M))$ is called regular if, for any vector $v \in E_{h}(h \in M)$, there exists a vector field $X$ such that $v=X(h)$. 
Definition 5.2. The differential space $(L, \mathfrak{C}(L))$ is called a semimanifold if there exists a structure $\mathfrak{D}$ of a manifold on $L$ of some constant dimension, such that

1. $(L, \mathfrak{D})$ is a differential subspace of the differential space $(L, \mathfrak{C}(L))$ (not proper),

2. for any locally arcwise connected topological space $X$ and for a continuous mapping $f: X \rightarrow(L, \mathfrak{C}(L))$, the mapping $\bar{f}: X \rightarrow(L, \mathfrak{D}), x \mapsto f(x)$, is continuous.

The manifold $(L, \mathfrak{D})$ is called a leaf of the semimanifold $(L, \mathfrak{C}(L))$. The differential structure $\mathfrak{D}$ is uniquely determined.

Let $(L, \mathfrak{C}(L))$ be a semimanifold with the leaf $(L, \mathfrak{D})$. For any differential space $(X, \mathfrak{C})$ with a locally arcwise connected topology and for any smooth mapping $f:(X, \mathfrak{C}) \rightarrow$ $(L, \mathfrak{C}(L))$ such that $f[X] \subset L$, the mapping $\bar{f}:(X, \mathfrak{C}) \rightarrow(L, \mathfrak{D}), x \mapsto f(x)$ is smooth $[\mathrm{K} 1]$.

The Cartesian product of semimanifolds is a semimanifold.

Definition 5.3. By a foliated groupoid (F-groupoid) (see [L1], [L2]) we mean a groupoid in the category of differential spaces $(\Phi, \alpha, \beta, M, \cdot)$ in which:

FG1 for each $x \in M$, the differential spaces $\tilde{\Phi}_{x}$ and $\tilde{L}_{x}$ are semimanifolds (leaves of these spaces we will be denoted by $\Phi_{x}$ and $L_{x}$ respectively),

FG2 for each $x \in M$ the mappings $\beta_{x}: \Phi_{x} \rightarrow L_{x}$ are submersions,

FG3 the distribution $T^{\alpha} \Phi=\bigsqcup_{h \in \Phi} T_{h}\left(\Phi_{\alpha h}\right) \subset T \Phi$ has the following property: for any $h \in \Phi$ and $v \in T_{h}\left(\Phi_{\alpha h}\right)$ there exists a smooth right-invariant vector field $X$ on $\Phi$ such that $X(h)=v$.

Corollary 5.4. For the F-groupoid $\Phi$, the distribution $T^{\alpha} \Phi$ on $\Phi$ and the distribution $E$ on $M$ of all vectors tangent to all leaves $L_{x} \subset M$ are regular. In particular, the family of immersed submanifolds $L_{x}$ is a foliation with singularities in the sense of $P$. Stefan.

An important example of an F-groupoid is given by

THEOREM 5.5. The Cartesian product of F-groupoids is an F-groupoid.

Proposition 5.6. If $(\Phi, \alpha, \beta, M, \cdot)$ is an F-groupoid, then the system $\left(\Phi_{x}, \beta_{x}, L_{x}\right.$, $\left.G_{x}, \cdot\right)$ where $\Phi_{x}, L_{x}, G_{x}$ are leaves of semimanifolds $\tilde{\Phi}_{x}, \tilde{L}_{x}, \tilde{G}_{x}$ respectively, is a principal bundle. Moreover, for $x \in M$, we have $\mathfrak{g}_{1 x}:=T_{u_{x}} G_{x}=\operatorname{ker}\left(\beta_{x}\right)_{* u_{x}}$ and $\operatorname{Im}\left(\beta_{x}\right)_{* u_{x}}=$ $T_{x} L_{x}=E_{x}$. So we have the exact sequence of vector spaces $0 \hookrightarrow \mathfrak{g}_{1 x} \hookrightarrow T_{u_{x}}\left(\Phi_{x}\right) \rightarrow E_{x} \rightarrow 0$.

Due to the construction of a smooth algebroid of a smooth groupoid we build an algebroid of an F-groupoid. We put

$$
A(\Phi):=\bigcup_{x \in M} T_{u_{x}} \Phi_{x} \subset T \Phi, \quad p: A(\Phi) \rightarrow M, \quad p(v)=x \Longleftrightarrow v \in T_{u_{x}} \Phi_{x} .
$$

On the set $A(\Phi)$ we introduce the differential structure $\mathfrak{C}(A(\Phi))=(\mathfrak{C}(T \Phi))_{A(\Phi)}$.

The differential space obtained above is a proper differential subspace of the differential space $(T \Phi, \mathfrak{C}(T \Phi))$. Moreover, the mapping $p: A(\Phi) \rightarrow M$ is smooth.

Proposition 5.7. The system $\left(A(\Phi), p, M,\left\{A(\Phi)_{\mid x}\right\}_{x \in M}\right)$ is a vector semibundle. 
Proof. The differential space $(T M, \mathfrak{C}(T M))$ with the natural projection on $(M, \mathfrak{C}(M))$ forms a vector semibundle. Then the assertion follows from the construction of the space $A(\Phi)$ and lemma 3.4 .

The following theorem establishes a relation between the module $X^{R}(\Phi)$ and the set $\operatorname{Sec} A(\Phi)$ of all global cross-sections of the projection $p: A(\Phi) \rightarrow M$.

TheOREM 5.8. If $X \in X^{R}(\Phi)$, then $X^{0}: M \rightarrow A(\Phi), x \mapsto X\left(u_{x}\right)$, is a smooth crosssection of the projection $p$. Conversely, for any smooth cross-section $\eta: M \rightarrow A(\Phi)$ of $p$ there exists exactly one smooth right-invariant vector field $\eta^{\prime}$ on $\Phi$ such that $\left(\eta^{\prime}\right)^{0}=\eta$. Moreover, the mappings $X^{R}(\Phi) \ni X \mapsto X^{0} \in \operatorname{Sec} A(\Phi)$ and $\operatorname{Sec} A(\Phi) \ni \eta \mapsto \eta^{\prime} \in X^{R}(\Phi)$ establish a canonical isomorphism of the $C^{\infty}(M)$-modules $X^{R}(\Phi)$ and $\operatorname{Sec} A(\Phi)$.

Now, in the module $\operatorname{Sec} A(\Phi)$ we introduce some structure of a Lie algebra by the formula $\llbracket \xi, \eta \rrbracket:=\left(\left[\xi^{\prime}, \eta^{\prime}\right]\right)^{0}$ for $\xi, \eta \in \operatorname{Sec} A(\Phi)$. The pair $(\operatorname{Sec} A(\Phi), \llbracket \cdot, \cdot \rrbracket)$ forms an $\mathbb{R}$-Lie algebra. Moreover, the canonical isomorphism described above is an isomorphism of Lie algebras.

Define a mapping $\tilde{\beta}_{*}: A(\Phi) \rightarrow T M, v \mapsto \beta_{*}(v)$. Note that the diagram below is commutative:

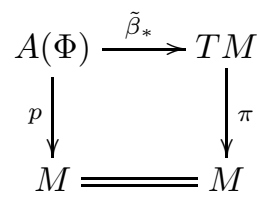

Then the mapping $\tilde{\beta}_{*}$ is a (strong) homomorphism of the vector semibundles $A(\Phi)$ and $T M$.

THEOREM 5.9. Any vector field $X \in X^{R}(\Phi)$ is $\beta$-projective, i.e. there exists exactly one vector field $Y \in X(M)$ with which $X$ is $\beta$-related. It is the field $Y:=\tilde{\beta}_{*} \circ\left(X^{0}\right)$.

Further, the field $\tilde{\beta}_{*} \circ \xi, \xi \in \operatorname{Sec} A(\Phi)$, will be briefly denoted by $\tilde{\beta}_{*}$. The following equality is true: $\xi^{\prime}(f \circ \beta)=\left(\beta_{*} \xi\right)(f) \circ \beta, \xi \in \operatorname{Sec} A(\Phi)$ and $f \in C^{\infty}(M)$. The following proposition gives an important property of the mapping $S e c \tilde{\beta}_{*}$.

Proposition 5.10. The mapping $\operatorname{Sec} \tilde{\beta}_{*}: \operatorname{Sec} A(\Phi) \rightarrow X(M), \xi \mapsto \beta_{*} \xi$ is a homomorphism of Lie algebras.

Proposition 5.11. For right-invariant vector fields $X, Y \in X^{R}(\Phi)$ and a function $f \in C^{\infty}(M),[X, f \circ \beta \cdot Y]=f \circ \beta \cdot[X, Y]+\left(\beta_{*} X^{0}\right)(f) \circ \beta \cdot Y$.

Corollary 5.12. The Lie algebra $\operatorname{Sec} A(\Phi)$ has the property $\llbracket \xi, f \cdot \eta \rrbracket=f \cdot \llbracket \xi, \eta \rrbracket+$ $\left(\beta_{*} \xi\right)(f) \cdot \eta$ for $\xi, \eta \in \operatorname{Sec} A(\Phi)$ and $f \in C^{\infty}(M)$.

Definition 5.13. By an F-algebroid over the differential manifold $M$ we mean the system

$$
\mathbf{A}=(A, \llbracket \cdot, \cdot \rrbracket, \gamma)
$$

in which:

(1) $A=\left(A, p, M,\left\{A_{\llcorner x}\right\}_{x \in M}\right)$ is a vector semibundle,

(2) $(\operatorname{Sec} A(\Phi), \llbracket \cdot, \cdot \rrbracket)$ is a Lie $\mathbb{R}$-algebra, 
(3) $\gamma: A \rightarrow T M$ is a (strong) semibundle homomorphism such that $\operatorname{Sec} \gamma: \operatorname{Sec} A(\Phi) \rightarrow$ $X(M)$ is a homomorphism of Lie algebras,

(4) for any cross-sections $\xi, \eta \in \operatorname{Sec} A(\Phi)$ and the mapping $f \in C^{\infty}(M)$, the equality $\llbracket \xi, f \cdot \eta \rrbracket=f \cdot \llbracket \xi, \eta \rrbracket+(\gamma \circ \xi)(f) \cdot \eta$ holds.

Clearly, $(\operatorname{Sec} A(\Phi))$ forms a Lie-Rinehart algebra.

EXAmple 1. For any F-groupoid $\Phi=(\Phi, \alpha, \beta, M, \cdot)$ the system $((A(\Phi), p, M)$, $\left.\llbracket \cdot, \cdot \rrbracket, \tilde{\beta}_{*}\right)$ is an F-algebroid. It is called the F-algebroid of the F-groupoid $\Phi$.

Definition 5.14. Let $A^{\prime}=\left(A^{\prime}, \llbracket \cdot, \cdot \rrbracket^{\prime}, \gamma^{\prime}\right)$ and $A=(A, \llbracket \cdot, \cdot \rrbracket, \gamma)$ be F-algebroids over the same differential manifold $M$. The mapping $H: A^{\prime} \rightarrow A$ is called a (strong) homomorphism of F-algebroids $A^{\prime}$ and $A$ if:

(i) $\mathrm{H}$ is a (strong) homomorphism of vector semibundles,

(ii) the mapping $\operatorname{SecH}: \operatorname{Sec} A^{\prime} \rightarrow \operatorname{Sec} A$ is a homomorphism of Lie algebras.

(iii) $\gamma \circ H=\gamma^{\prime}$.

Proposition 5.15. For any $x \in M$, the pair $\left(\mathfrak{g}_{1 x},[\cdot, \cdot]\right)$ is a Lie algebra called the isotropy algebra at the point $x$ with the commutator defined by $[v, w]:=\llbracket \xi, \eta \rrbracket(x)$ for $\xi, \eta \in \operatorname{Sec} A$ such that $\xi(x)=v$ and $\eta(x)=w, v, w \in \mathfrak{g}_{1 x}$. Moreover, the Lie algebra $\mathfrak{g}_{1 x}$ is the right Lie algebra of the Lie group $G_{x}$.

Definition 5.16. By the derivative of the representation $T: \Phi * \mathfrak{f} \rightarrow \mathfrak{f}$ of the Fgroupoid $\Phi$ in the semibundle $\mathfrak{f}$ we mean the $C^{\infty}(M)$-linear homomorphism of Lie algebras $T^{\prime}: X^{R}(\Phi) \rightarrow C D O(\mathfrak{f})$, which is the restriction of $\tilde{T}^{\prime}: \tilde{X}^{R}(\Phi) \rightarrow X(M)$ to the subalgebra $X^{R}(\Phi)$.

Of course, the following equality holds: $T^{\prime}(\xi)(\sigma)=\xi\left(\sigma^{T}\right) \circ u$.

Definition 5.17. By the inner representation of the F-groupoid $\Phi$ we mean the mapping Ad: $\Phi * \mathfrak{g} \rightarrow \mathfrak{g}$ defined by the formula $A d(h)=\left(\tau_{h}\right)_{* u_{x}}: \mathfrak{g}_{1 x} \rightarrow \mathfrak{g}_{1 y}$ where $x=\alpha h, y=\beta h, \tau_{h}: G_{x} \rightarrow G_{y}, a \mapsto h \cdot a \cdot h^{-1}$, i.e. such that the following diagram commutes

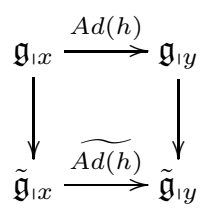

The smoothness of $A d$ follows from the smoothness of $\widetilde{A d(h)}$.

LEMMA 5.18. Right-invariant vector fields on $\Phi$ restricted to the manifold $\Phi_{x}$ generate the module of all smooth vector fields on $\Phi_{x}$.

6. The Cartesian product of F-algebroids. Consider the Cartesian product of differential manifolds $M^{1} \times M^{2}$. We identify $T_{\left(x_{1}, x_{2}\right)}\left(M^{1} \times M^{2}\right)=T_{x_{1}} M^{1} \times T_{x_{2}} M^{2}$.

Let $\bar{X}=\left(X^{1}, X^{2}\right), \bar{Y}=\left(Y^{1}, Y^{2}\right) \in X\left(M^{1} \times M^{2}\right)$ be two vector fields tangent to the Cartesian product $M^{1} \times M^{2}$. The decomposition of the vector field $[\bar{X}, \bar{Y}]$ to the part $[\bar{X}, \bar{Y}]^{1}$ tangent to $M^{1}$ and the part $[\bar{X}, \bar{Y}]^{2}$ tangent to $M^{2}$ is given by the formulae

$$
[\bar{X}, \bar{Y}]_{\left(x_{1}, x_{2}\right)}^{1}=\left[X^{1}\left(\cdot, x_{2}\right), Y^{1}\left(\cdot, x_{2}\right)\right]\left(x_{1}\right)+X_{\left(x_{1}, x_{2}\right)}^{2}\left(Y^{1}\left(x_{1}, \cdot\right)\right)-Y_{\left(x_{1}, x_{2}\right)}^{2}\left(X^{1}\left(x_{1}, \cdot\right)\right),
$$




$$
[\bar{X}, \bar{Y}]_{\left(x_{1}, x_{2}\right)}^{2}=\left[X^{1}\left(\cdot, x_{2}\right), Y^{1}\left(\cdot, x_{2}\right)\right]\left(x_{1}\right)+X_{\left(x_{1}, x_{2}\right)}^{2}\left(Y^{1}\left(x_{1}, \cdot\right)\right)-Y_{\left(x_{1}, x_{2}\right)}^{2}\left(X^{1}\left(x_{1}, \cdot\right)\right) .
$$

where $X_{\left(x_{1}, x_{2}\right)}^{1} \in T_{x_{1}} M^{1}$ and $X_{\left(x_{1}, x_{2}\right)}^{2} \in T_{x_{2}} M^{2}$.

THEOREM 6.1. Let $\Phi_{1}, \Phi_{2}$ be F-groupoids which algebroids are $\left(A\left(\Phi_{1}\right), \llbracket \cdot, \cdot \rrbracket,\left(\tilde{\beta}_{1}\right)_{*}\right)$ and $\left(A\left(\Phi_{2}\right), \llbracket \cdot, \cdot \rrbracket,\left(\tilde{\beta}_{2}\right)_{*}\right)$, respectively. Then the F-algebroid of the Cartesian product $\Phi_{1} \times$ $\Phi_{2}$ is $\left(A\left(\Phi_{1}\right) \times A\left(\Phi_{2}\right), \llbracket \cdot, \cdot \rrbracket,\left(\tilde{\beta}_{1}\right)_{*} \times\left(\tilde{\beta}_{2}\right)_{*}\right)$, where $A\left(\Phi_{1}\right) \times A\left(\Phi_{2}\right)$ is a Cartesian product of vector semibundles and, for any cross-sections $\bar{\xi}=\left(\xi^{1}, \xi^{2}\right), \bar{\eta}=\left(\eta^{1}, \eta^{2}\right)$ of the projection $p_{1} \times p_{2}$, the commutator $\llbracket \cdot, \cdot \rrbracket=\left(\llbracket \cdot, \cdot \rrbracket^{1}, \llbracket \cdot, \cdot \rrbracket^{2}\right)$ is given by the formulae

$$
\begin{aligned}
\llbracket \bar{\xi}, \bar{\eta} \rrbracket_{\left(x_{1}, x_{2}\right)}^{1}= & \llbracket \xi^{1}\left(\cdot, x_{2}\right), \eta^{1}\left(\cdot, x_{2}\right) \rrbracket\left(x_{1}\right)+\beta_{2 *}\left(\left(\xi^{2}\right)_{\left(x_{1}, x_{2}\right)}\left(\eta^{1}\left(x_{1}, \cdot\right)\right)\right) \\
& -\beta_{2 *}\left(\eta^{2}\right)_{\left(x_{1}, x_{2}\right)}\left(\eta^{1}\left(x_{1}, \cdot\right)\right), \\
\llbracket \bar{\xi}, \bar{\eta} \rrbracket_{\left(x_{1}, x_{2}\right)}^{2}= & \llbracket \xi^{2}\left(x_{1}, \cdot\right), \eta^{2}\left(x_{1}, \cdot\right) \rrbracket\left(x_{2}\right)+\beta_{1 *}\left(\left(\xi^{1}\right)_{\left(x_{1}, x_{2}\right)}\left(\eta^{2}\left(\cdot, x_{2}\right)\right)\right) \\
& -\beta_{1 *}\left(\eta^{1}\right)_{\left(x_{1}, x_{2}\right)}\left(\eta^{2}\left(\cdot, x_{2}\right)\right) .
\end{aligned}
$$

This algebroid will be denoted briefly by $A\left(\Phi_{1}\right) \times A\left(\Phi_{2}\right)$.

7. Cohomology with coefficients of F-groupoids. Let $\Phi$ and $\mathfrak{f}$ be an F-groupoid and a vector semibundle, both over the same manifold $M$. In the vector space $\bigwedge T_{h}^{*} \Phi_{\alpha h} \otimes$ $\mathfrak{f}_{1 \alpha h}$ there exists a structure of a left module over the algebra $\bigwedge T_{h}^{*} \Phi_{\alpha h}$, defined uniquely by the formula $\phi \wedge(\psi \otimes v):=(\phi \wedge \psi) \otimes v$ for $\phi, \psi \in \wedge T_{h}^{*} \Phi_{\alpha h}$ and $v \in \mathfrak{f}_{1 \alpha h}$.

The canonical duality $\langle\cdot, \cdot\rangle: \bigwedge T_{h}^{*} \Phi_{\alpha h} \times \bigwedge T_{h} \Phi_{\alpha h} \rightarrow \mathbb{R}$ uniquely determines a $2-\mathbb{R}$ linear mapping $\langle\cdot, \cdot\rangle:\left(\bigwedge T_{h}^{*} \Phi_{\alpha h} \otimes \mathfrak{f}_{1 \alpha h}\right) \times \bigwedge T_{h} \Phi_{\alpha h} \rightarrow \mathfrak{f}_{1 \alpha h}$ by the formula $\langle\phi \otimes v, \psi\rangle=$ $\langle\phi, \psi\rangle \otimes v$ for $\phi, \psi \in \bigwedge T_{h}^{*} \Phi_{\alpha h}$ and $v \in \mathfrak{f}_{1 \alpha h}$.

Definition 7.1. An $\alpha$-form of order $q$ on $\Phi$ with values in $\mathfrak{f}$ is a mapping $\Psi$ which assigns a covector $\Psi(h) \in \bigwedge^{q} T_{h}^{*} \Phi_{\alpha h} \otimes \mathfrak{f}_{1 \alpha h}$ to each element $h \in \Phi$.

Definition 7.2. The $\alpha$-form $\Psi$ of order $q$ on $\Phi$ with values in $\mathfrak{f}$ will be called smooth if for any right-invariant vector fields $\xi_{1}, \ldots, \xi_{q}$ on the F-groupoid $\Phi$, the mapping $\Phi\left(\xi_{1}, \ldots, \xi_{q}\right): \Phi \rightarrow \mathfrak{f}, h \mapsto\left\langle\Psi(h), \xi_{1}(h) \wedge \ldots \wedge \xi_{q}(h)\right\rangle$, is smooth.

If $\mathfrak{f}=M \times \mathbb{R}$ is a trivial linear bundle of rank 1 , then $\Psi$ will be called a (real) $\alpha$-form of order $q$ on the F-groupoid $\Phi$. The set $\Omega^{\alpha}(\Phi ; \mathfrak{f})$ of all smooth $\alpha$-forms on $\Phi$ with values in the semibundle $\mathfrak{f}$ forms a left graded $\mathfrak{C}(\mathfrak{f})$-module and a left module over the algebra $\Omega^{\alpha}(\Phi)$ of all smooth real $\alpha$-forms on $\Phi$.

Let $i_{x}: \Phi_{x} \rightarrow \Phi$ denote an inclusion for $x \in M$. It is a smooth mapping allowing to pull back the $\alpha$-forms $\Psi \in \Omega^{\alpha}(\Phi ; \mathfrak{f})$. For a $q$-form $\Psi \in \Omega^{\alpha, q}(\Phi ; \mathfrak{f}), \Psi_{1 x}:=i_{x}^{*}(\Psi)$ is the $q$-form on $\Phi_{x}$ defined by the formula $i_{x}^{*}(\Psi)(h)=\Psi(h) \in \bigwedge^{q} T_{h}^{*} \Phi_{x} \otimes \mathfrak{f}_{x x}$. Since the right-invariant vector fields on $\Phi_{x}$ generate the whole module of smooth vector fields on $\Phi_{x}$, then $i_{x}^{*}(\Psi)$ is a $q$-form on $\Phi_{x}$ smooth in the ordinary sense. For $\xi_{1}, \ldots, \xi_{q} \in \mathfrak{X}^{R}(\Phi)$, we have

$$
i_{x}^{*}(\Psi)\left(\xi_{1}\left|\Phi_{x}, \ldots, \xi_{q}\right| \Phi_{x}\right)=\left(\Psi\left(\xi_{1}, \ldots, \xi_{q}\right)\right) \mid \Phi_{x} .
$$

Definition 7.3. Let $T$ be a representation of the groupoid $\Phi$ in the semibundle $\mathfrak{f}$. An $\alpha$-form $\Psi \in \Omega^{\alpha}(\Phi ; \mathfrak{f})$ will be called $T$-equivariant if $\bigwedge_{h \in \Phi}\left(D_{h}^{*}\right)\left(\Psi_{\llcorner\alpha h}\right)=T\left(h^{-1}\right)_{*}\left(\Psi_{\text {। } \beta h}\right)$.

LEMMA 7.4. An $\alpha$-form $\Psi$ of order $q$ on the F-groupoid $\Phi$ is $T$-equivariant if and only if, for any right-invariant vector fields $\xi_{1}, \ldots, \xi_{q} \in X^{R}(\Phi)$ the mapping $\Psi\left(\xi_{1}, \ldots, \xi_{q}\right)$ has 
the form $\sigma^{T}$ for some cross-section $\sigma$ of the semibundle $\mathfrak{f}$, i.e. $\Psi\left(\xi_{1}, \ldots, \xi_{q}\right)(h)=\sigma^{T}(\beta h)$ for $h \in \Phi$.

LEMma 7.5. A T-equivariant $\alpha$-form $\Psi$ is smooth if and only if, for any crosssection $\xi_{1}^{0}, \ldots, \xi_{q}^{0} \in \operatorname{Sec} A(\Phi)$ the cross-section $\sigma: M \rightarrow \mathfrak{f}$ defined by the formula $\sigma(x)=$ $\left\langle\Psi_{u_{x}}, \xi_{1 x}^{0} \wedge \ldots \wedge \xi_{q x}^{0}\right\rangle$ is smooth.

The real forms equivariant with respect to the trivial representation $T(h)=i d_{\mathbb{R}}$ are usual right-invariant forms, i.e. such that $D_{h}^{*}\left(\psi_{1 \alpha h}\right)=\psi_{\text {เ } \beta h}$ for $h \in \Phi$. The set of all such forms will be denoted by $\Omega_{\mathbb{R}}^{\alpha}(\Phi)$. The vector space of all $T$-equivariant $\alpha$-forms on $\Phi$ with values in $\mathfrak{f}$ will be denoted by $\Omega_{T}^{\alpha}(\Phi ; \mathfrak{f})$.

LEMMA 7.6. The space $\Omega_{T}^{\alpha}(\Phi ; \mathfrak{f})$ is

1. a graded module over the ring $C^{\infty}(M)$, with respect to the multiplication $f \bullet \Psi:=$ $f \circ \beta \cdot \Psi$.

2. a module over the algebra $\Omega_{\mathbb{R}}^{\alpha}(\Phi)$ of all equivariant $\alpha$-forms on $\Phi$.

Now let $A=(A, \llbracket \cdot, \cdot \rrbracket, \gamma)$ be an algebroid over the foliated manifold $M$ where $A$ is a vector semibundle over $M$, and the anchor $\gamma: A \rightarrow E$ is an epimorphism on the regular distribution $E$. The mapping $\vartheta$ assigning to the point $x \in M$ the covector $\vartheta(x) \in$ $\bigwedge^{q} A_{1 x}^{*} \otimes \mathfrak{f}_{1 x}$ will be called smooth if the mapping $x \mapsto\left\langle\vartheta(x), \xi_{1 x} \wedge \ldots \wedge \xi_{q x}\right\rangle$ is smooth, where $\xi_{1}, \ldots, \xi_{q}$ are right-invariant vector fields on the F-groupoid $\Phi$.

Denote $\Omega_{A(\Phi)}(M ; \mathfrak{f}):=\bigoplus_{q \geq 0} \Omega_{A(\Phi)}^{q}(M ; \mathfrak{f})$ where $\Omega_{A(\Phi)}^{q}(M ; \mathfrak{f})$ consists of mappings $\vartheta$ assigning to each point $x \in M$ a covector $\vartheta(x) \in \bigwedge^{q} A_{1 x}^{*} \otimes \mathfrak{f}_{1 x}$. In particular, for $\mathfrak{f}=M \times \mathbb{R}, \Omega_{A}(M)=\bigoplus_{q \geq 0} \Omega_{A}^{q}(M)$ where $\Omega_{A}^{q}(M)$ consists of the smooth mappings $\vartheta=\left(x \mapsto \vartheta(x) \in \bigwedge^{q} A_{1 x}^{*}\right)$.

TheOREM 7.7. The mapping $\tau_{T}: \Omega_{T}^{\alpha}(\Phi ; \mathfrak{f}) \rightarrow \Omega_{A(\Phi)}(M ; \mathfrak{f})$, defined by the formula $\tau_{T}(\Psi)(x)=\Psi\left(u_{x}\right), \quad x \in M$, is an isomorphism of $C^{\infty}(M)$-modules.

(a) Let $X$ be any right-invariant vector field on the groupoid $\Phi$. There exist endomorphisms $i_{X}^{\alpha, \mathfrak{f}}, d^{\alpha, \mathfrak{f}}, \theta_{X}^{\alpha, \mathfrak{f}}$ of the vector space $\Omega^{\alpha}(\Phi ; \mathfrak{f})$ defined uniquely by the condition: for any $x \in M$, the diagram below commutes:

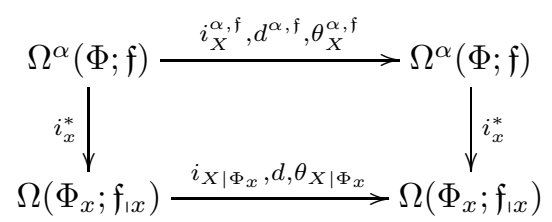

(b) These endomorphisms are defined by the global formulae:

1. $\left(i_{X}^{\alpha, \mathfrak{f}} \Psi\right)\left(X_{1}, \ldots, X_{q-1}\right)=\Psi\left(X, X_{1}, \ldots, X_{q-1}\right)$,

2. $\left(\theta_{X}^{\alpha, \mathfrak{f}} \Psi\right)\left(X_{1}, \ldots, X_{q}\right)=X\left(\Psi\left(X_{1}, \ldots, X_{q}\right)\right)-\sum_{j=1}^{q} \Psi\left(X_{1}, \ldots,\left[X, X_{j}\right], \ldots, X_{q}\right)$,

3. $\left(d^{\alpha, \mathfrak{f}} \Psi\right)\left(X_{0}, \ldots, X_{q}\right)=\sum_{j=1}^{q}(-1)^{j} X_{j}\left(\Psi\left(X_{0}, \ldots, \hat{X}_{j}, \ldots, X_{q}\right)\right)$

$+\sum_{i<j}(-1)^{i+j} \Psi\left(\left[X_{i}, X_{j}\right], \ldots, \hat{X}_{i}, \ldots, \hat{X}_{j}, \ldots, X_{q}\right)$,

where $\Psi \in \Omega^{\alpha, q}(\Phi ; \mathfrak{f})$, and $X_{1}, \ldots, X_{q} \in \mathfrak{X}^{R}(\Phi)$.

(c) For any representation $T$ of the groupoid $\Phi$ in the semibundle $\mathfrak{f}$, the subspace $\Omega_{T}^{\alpha}(\Phi ; \mathfrak{f})$ is stable with respect to the above endomorphisms. 
(d) For $\psi \in \Omega^{\alpha, q}(\Phi ; \mathfrak{f}), \Psi \in \Omega^{\alpha}(\Phi ; \mathfrak{f})$ and a field $X \in \mathfrak{X}^{R}(\Phi)$ we have:
1. $i_{X}^{\alpha, \mathfrak{f}}(\psi \wedge \Psi)=i_{X}^{\alpha, \mathfrak{f}} \psi \wedge \Psi+(-1)^{q} \psi \wedge i_{X}^{\alpha, \mathfrak{f}} \Psi$,
2. $\theta_{X}^{\alpha, \mathfrak{f}}(\psi \wedge \Psi)=\theta_{X}^{\alpha, \mathfrak{f}} \psi \wedge \Psi+\psi \wedge \theta_{X}^{\alpha, \mathfrak{f}} \Psi$,
3. $d^{\alpha, \mathfrak{f}}(\psi \wedge \Psi)=d^{\alpha, \mathfrak{f}} \psi \wedge \Psi+(-1)^{q} \psi \wedge d^{\alpha, \mathfrak{f}} \Psi$,
4. $i_{[X, Y]}^{\alpha, \mathfrak{f}}=i_{X}^{\alpha, \mathfrak{f}} \circ i_{Y}^{\alpha, f}-i_{Y}^{\alpha, \mathfrak{f}} \circ i_{X}^{\alpha, f}$,
5. $\theta_{[X, Y]}^{\alpha, \mathfrak{f}}=\theta_{X}^{\alpha, \mathfrak{f}} \circ \theta_{Y}^{\alpha, \mathfrak{f}}-\theta_{Y}^{\alpha, \mathfrak{f}} \circ \theta_{X}^{\alpha, \mathfrak{f}}$,
6. $\theta_{X}^{\alpha, \mathfrak{f}}=\theta_{X}^{\alpha, \mathfrak{f}} \circ d^{\alpha, \mathfrak{f}}+d^{\alpha, \mathfrak{f}} \circ i_{X}^{\alpha, \mathfrak{f}}$,
7. $d^{\alpha, \mathfrak{f}} \circ d^{\alpha, \mathfrak{f}} 0$,
8. $d^{\alpha, \mathfrak{f}} \circ \theta_{X}^{\alpha, \mathfrak{f}}=\theta_{X}^{\alpha, \mathfrak{f}} \circ d^{\alpha, \mathfrak{f}}$.

Definition 7.8. For $\xi \in \operatorname{Sec} A(\Phi)$ define the endomorphisms $i_{\xi}^{A, \mathfrak{f}}, d^{A, \mathfrak{f}}$, and $\theta_{\xi}^{A, \mathfrak{f}}$ of the space $\Omega_{A(\Phi)}(M ; \mathfrak{f})$ such that the diagram below commutes

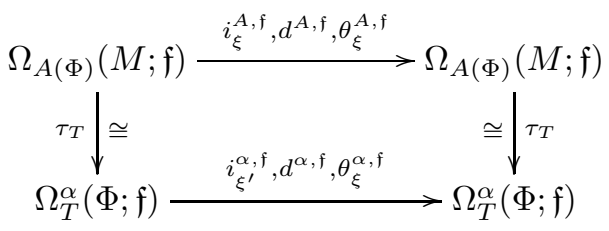

In the diagram above $\xi^{\prime}$ denotes the right-invariant vector field on the groupoid $\Phi$ generated by the cross-section $\xi \in \operatorname{Sec} A(\Phi)$.

THEOREM 7.9. (a) The endomorphisms $i_{\xi}^{A, \mathfrak{f}}, d^{A, \mathfrak{f}}$, and $\theta_{\xi}^{A, \mathfrak{f}}$ have the properties:

1. $i_{\xi}^{A, \mathfrak{f}}(\psi \wedge \Psi)=i_{\xi}^{A}(\psi) \wedge \Psi+(-1)^{q} \psi \wedge i_{\xi}^{A, \mathfrak{f}}(\Psi)$,

2. $\theta_{\xi}^{A, \mathfrak{f}}(\psi \wedge \Psi)=\theta_{\xi}^{A}(\psi) \wedge \Psi+\psi \wedge \theta_{\xi}^{A, \mathfrak{f}}(\Psi)$,

3. $d^{A, \mathfrak{f}}(\psi \wedge \Psi)=d^{A}(\psi) \wedge \Psi+(-1)^{q} \psi \wedge d^{A, \mathfrak{f}}(\Psi)$,

4. $i_{[\xi, \eta]}^{A, \mathfrak{f}}=i_{\xi}^{A, \mathfrak{f}} \circ i_{\eta}^{A, \mathfrak{f}}-i_{\eta}^{A, \mathfrak{f}} \circ i_{\xi}^{A, \mathfrak{f}}$,

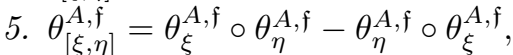

6. $\theta_{\xi}^{A, \mathfrak{f}}=i_{\xi}^{A, \mathfrak{f}} \circ d^{A, \mathfrak{f}}+d^{A, \mathfrak{f}} \circ i_{\xi}^{A, \mathfrak{f}}$,

7. $d^{A, \mathfrak{f}} \circ d^{A, \mathfrak{f}}=0$,

8. $d^{A, \mathfrak{f}} \circ \theta_{\xi}^{A, \mathfrak{f}}=\theta_{\xi}^{A, \mathfrak{f}} \circ d^{A, \mathfrak{f}}$.

(b) The above endomorphisms are defined by the global formulae:

1. $\left(i_{\xi}^{A, \mathfrak{f}} \Psi\right)\left(\xi_{1}, \ldots, \xi_{q-1}\right)=\Psi\left(\xi, \xi_{1}, \ldots, \xi_{q-1}\right)$,

2. $\left(\theta_{\xi}^{A, \mathfrak{f}} \Psi\right)\left(\xi_{1}, \ldots, \xi_{q}\right)=T^{\prime}\left(\xi^{\prime}\right)\left(\Psi\left(\xi_{1}, \ldots, \xi_{q}\right)\right)-\sum_{j=1}^{q} \Psi\left(\xi_{1}, \ldots, \llbracket \xi, \xi_{j} \rrbracket, \ldots \xi_{q}\right)$,

3. $\left(d^{A, \mathfrak{f}} \Psi\right)\left(\xi_{0}, \ldots, \xi_{q}\right)=\sum_{j=1}^{q}(-1)^{j} T^{\prime}\left(\xi_{j}\right)\left(\Psi\left(\xi_{0}, \ldots, \hat{\xi}_{j}, \ldots, \xi_{q}\right)\right)$

$+\sum_{i<j}(-1)^{i+j} \Psi\left(\llbracket \xi_{i}, \xi_{j} \rrbracket, \ldots, \hat{\xi}_{i}, \ldots, \hat{\xi}_{j}, \ldots, \xi_{q}\right)$

(c) In particular, for real forms and the trivial representation $T$ in the bundle $M \times \mathbb{R}$ (then $\left.T^{\prime}(\xi)=\gamma \circ \xi\right)$ we have:

1. $\left(\theta_{\xi}^{A} \Psi\right)\left(\xi_{1}, \ldots, \xi_{q}\right)=(\gamma \circ \xi)\left(\Psi\left(\xi_{1}, \ldots, \xi_{q}\right)\right)-\sum_{j=1}^{q} \Psi\left(\xi_{1}, \ldots, \llbracket \xi, \xi_{j} \rrbracket, \ldots \xi_{q}\right)$,

2. $\left(d^{A} \Psi\right)\left(\xi_{0}, \ldots, \xi_{q}\right)=\sum_{j=1}^{q}(-1)^{j}\left(\gamma \circ \xi_{j}\right)\left(\Psi\left(\xi_{0}, \ldots, \hat{\xi}_{j}, \ldots, \xi_{q}\right)\right)$

$+\sum_{i<j}(-1)^{i+j} \Psi\left(\llbracket \xi_{i}, \xi_{j} \rrbracket, \ldots, \hat{\xi}_{i}, \ldots, \hat{\xi}_{j}, \ldots, \xi_{q}\right)$. 
8. Connections. Fix an F-groupoid $\Phi$ over the foliated manifold $(M, E)$ and its algebroid $(A(\Phi), \llbracket \cdot, \cdot \rrbracket, \gamma)$.

Definition 8.1. By a connection in the F-groupoid $\Phi$ over the foliated manifold $(M, E)$ we mean a regular distribution $H \subset T^{\alpha} \Phi$ on the differential space $\Phi$, such that:

1. $\left(D_{h}\right)_{*}\left[H_{g}\right]=H_{g h}$ for $(g, h) \in \Phi * \Phi$,

2. $T_{h}\left(\Phi_{\alpha h}\right)=\mathfrak{g}_{1 h}^{\alpha} \oplus H_{h}$ where $\mathfrak{g}_{1 h}^{\alpha}=\operatorname{ker}\left(\beta_{1 \alpha h}\right)_{* h}$ is the vertical subspace of the space $T_{h}\left(\Phi_{\alpha h}\right)$,

3. for any smooth vector field $X \in X(F)$, its horizontal lifting $X^{H} \in X(H)$ defined by the conditions $X^{H}(h) \in T_{h}^{\alpha} \Phi$ and $\left(\left(\beta_{1 \alpha h}\right)_{* h}\right) X^{H}(h)=X(\beta h)$ is smooth.

The connection $H$ restricted to the principal bundle $\Phi_{x}$ is a connection in the usual sense.

Take any transitive algebroid $A$ over the manifold $M$, together with the short exact Atiyah sequence $0 \rightarrow \operatorname{ker} \gamma \hookrightarrow A \stackrel{\gamma}{\rightarrow} T M \rightarrow 0$, and take any foliation $E$ with singularities in the sense of P. Stefan. Define the algebroid $A^{\prime}$ as the inverse image of the foliation $E$ by the mapping $\gamma$, i.e. $A^{\prime}=\gamma^{-1}(E)$. Since the dimensions of fibres over different points of the distribution are different, the algebroid $A^{\prime}$ is not regular. $A^{\prime}$ is not a vector bundle. The sequence $0 \rightarrow \operatorname{ker} \gamma \hookrightarrow A^{\prime} \rightarrow E \rightarrow 0$ has, as a splitting, the restriction of any connection in the algebroid $A$. Of course, it is a connection in $A^{\prime}$.

It is easy to see that if the foliation $E$ does not have a constant rank and $A$ is not a vector bundle, then such a splitting does not exist. We shall consider only groupoids in which there exists a connection. Let $H$ be a connection in the groupoid $\Phi$. Define the mapping $\lambda: E \rightarrow A(\Phi)$ by the formula $\lambda(v)=\left(\gamma_{x} \mid H_{u_{x}}\right)^{-1}(v)$ for $v \in E_{x}, x \in M$.

THEOREM 8.2. Let $H$ be any connection in the F-groupoid $\Phi$. The mapping $\lambda: E \rightarrow$ $A(\Phi)$ defined above has the following properties:

1. $\lambda \mid E_{1 x}: E_{\mid x} \rightarrow A(\Phi)_{1 x}$ is a linear mapping,

2. for any vector field $X \in \mathfrak{X}(E), \lambda \circ X$ is a smooth cross-section of the vector semibundle $A(\Phi)$,

3. $\gamma \circ \lambda=i d$.

Conversely, any mapping $\lambda: E \rightarrow A(\Phi)$ which has the above properties uniquely determines some connection in the F-groupoid $\Phi$.

Proof. $\Leftarrow$ Let $\lambda: E \rightarrow A(\Phi)$ be a mapping fulfilling the above three conditions of the theorem. First, we show that $\lambda$ determines a regular distribution. The mapping $\lambda$ determines, for any $h \in \Phi$, the subspace $H_{h} \subset T_{h}^{\alpha} \Phi=T_{h} \Phi_{\alpha h}$ such that $H_{h}=\left(D_{h}\right)_{* \beta h}\left[\operatorname{Im} \lambda_{\beta h}\right]$, so it determines some regular distribution $H=\bigcup_{h \in \Phi} H_{h} \subset T^{\alpha} \Phi$.

Now, we show that the distribution $H$ fulfils conditions (1)-(3) from the definition of a connection.

(1) Let $(g, h) \in \Phi * \Phi$. From the definition we have $H_{g}=\left(D_{h}\right)_{* u_{\beta h}}\left[\operatorname{Im} \lambda_{\beta h}\right]$, then $\left(D_{h}\right)_{*}\left[H_{h}\right]=H_{g h}$.

(2) Note that $\operatorname{dimg} \mathfrak{g}_{1 h}^{\alpha}+\operatorname{dim} H_{h}=\operatorname{dim}\left(\operatorname{ker}\left(\beta_{1 \alpha h}\right)_{* h}\right)+\operatorname{dim}\left(D_{g}\right)_{* \beta g}\left[\operatorname{Im} \lambda_{\beta g}\right]=\operatorname{dim} T_{h}\left(\Phi_{\alpha h}\right)$ and $\mathfrak{g}_{1 h}^{\alpha} \cap H_{h}=\operatorname{ker}\left(\beta_{1 \alpha h}\right)_{* h} \cap\left(D_{g}\right)_{* \beta g}\left[\operatorname{Im} \lambda_{\beta g}\right]=\emptyset$. So, $T_{h}\left(\Phi_{\alpha h}\right)=\mathfrak{g}_{1 h}^{\alpha} \oplus H_{h}$. 
(3) Since $(\lambda \circ X)^{\prime}$ is a smooth vector field, therefore, for any vector field $X \in \mathfrak{X}(E)$, the smoothness of its horizontal lifting follows from the above lemma.

Lemma 8.3. Let $X \in \mathfrak{X}(E)$. Then $X^{H}=(\lambda \circ X)^{\prime}$.

Proof. For any $h \in \Phi$ we have

$$
\begin{aligned}
X^{H}(h) & =\left(\left(\beta_{y}\right)_{* h} \mid H_{y}\right)^{-1}\left(X_{x}\right)=\left(D_{h}\right)_{* u_{y}} \circ\left(\left(\left(\beta_{y}\right)_{* u_{y}} \mid H_{u_{y}}\right)^{-1}\right)\left(X_{y}\right) \\
& =\left(D_{h}\right)_{* u_{y}}\left((\lambda \circ X)_{u_{y}}^{\prime}\right)=(\lambda \circ X)_{h}^{\prime} .
\end{aligned}
$$

$\Rightarrow$ Follows from the linearity of $\gamma_{x}$, and from the smoothness of the horizontal lifting and the fact that the diagram below commutes

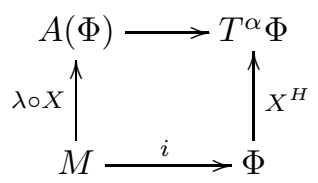

Definition 8.4. The mapping $\lambda: E \rightarrow A(\Phi)$ defined in theorem 8.2 will be called a connection in the algebroid $A(\Phi)$.

The theorem below shows an obstacle to the existence of a connection.

THEOREM 8.5. If there exists a connection in the groupoid $\Phi$, then the sequence $A(\Phi) \underset{S e c \gamma}{\longrightarrow} \operatorname{Sec} E=X(E) \rightarrow 0$ is exact.

REMARK 8.6. If $A(\Phi)$ is a vector bundle over the manifold $M$ and $E \subset T M$ is a foliation with singularities in the sense of P. Stefan, then the connection does not exist in such an algebroid. This is the reason to use the notion of a semibundle to define an F-algebroid.

Definition 8.7. Let $\lambda: E \rightarrow A(\Phi)$ be the connection in the algebroid $A(\Phi)$ and $\mathfrak{g}=$ ker $\gamma$ be a semibundle. By the connection form of the connection $\lambda$ we mean the mapping $\omega: A(\Phi) \rightarrow \mathfrak{g}$ uniquely defined by the conditions:

1. $\omega \mid \mathfrak{g}=i d$,

2. $k e r \omega=\operatorname{Im} \lambda$.

THEOREM 8.8. (a) If $\lambda$ is a connection in the F-algebroid $A(\Phi)$, then its connection form $\omega$ fulfils the condition:

$\left(^{*}\right)$ for any cross-section $\xi \in \operatorname{Sec} A(\Phi)$ the mapping $\omega \circ \xi$ is smooth.

(b) If for the F-algebroid $A(\Phi)$ the sequence $A(\Phi) \underset{\text { Sec } \gamma}{\longrightarrow} \operatorname{Sec} E=X(E) \rightarrow 0$ is exact then the mapping $\omega: A(\Phi) \rightarrow \mathfrak{g}$ is the connection form of some connection if and only if it fulfils the condition $\left({ }^{*}\right)$ and the equality $\omega \mid \mathfrak{g}=i d$.

Proof. (a) Take some cross-section $\xi \in \operatorname{Sec} A(\Phi)$. Then $\gamma \circ \xi \in \operatorname{SecE}$. Let $\xi=\xi_{1}+\lambda \circ$ $(\gamma \circ \xi)$ where $\xi_{1}$ is the horizontal part and $\lambda \circ(\gamma \circ \xi)$ is the vertical part of $\xi$. Since $\xi_{1}$ is smooth and $\xi_{1}=\xi-\lambda(\gamma \circ \lambda) \in \operatorname{Secg}$ then $\omega(\xi)=\xi-\lambda(\gamma \circ \lambda) \in \operatorname{Sec} \mathfrak{g}$.

(b) Assume that the mapping $\omega: A(\Phi) \rightarrow \mathfrak{g}$ fulfils (1) and (2). According to theorem 8.2 it is enough to construct a mapping $\lambda: E \rightarrow A(\Phi)$ linear on the fibres of semibundles, right-inverse to $\gamma$ and such that, for any vector field $X \in X(E)$ tangent to the foliation 
$E$, the field $\lambda \circ X$ is smooth. Put $\lambda(v)=\left(\gamma_{x} \mid \operatorname{ker} \omega_{x}\right)^{-1}(v)$ for $v \in E_{1 x}, x \in M$. The mapping $\lambda$ is linear on the fibres and $\gamma \circ \lambda=i d$. Now, take some vector field $X \in X(E)$. The smoothness of the field $\lambda \circ X$ follows from the lemma below.

Lemma 8.9. Let $\lambda: E \rightarrow A(\Phi)$ be the mapping defined above. For any vector field $X \in S e c E$ the field $\lambda \circ X$ is smooth if and only if the sequence $A(\Phi) \underset{\operatorname{Sec} \gamma}{\longrightarrow} \operatorname{Sec} E=X(E) \rightarrow 0$ is exact.

Consider the mapping $\gamma^{\alpha}: T^{\alpha} \Phi \rightarrow \Phi \times E, v \mapsto\left(\pi^{\alpha} v, \beta_{*} v\right)$ where $\pi^{\alpha}: T^{\alpha} \Phi \rightarrow \Phi$ is the natural projection. Denote $\mathfrak{g}^{\alpha}=\operatorname{ker} \gamma^{\alpha}$. From lemma 3.4 it follows that $\mathfrak{g}^{\alpha}$ is a vector semibundle.

Definition 8.10. Let $H$ be a connection in the F-groupoid $\Phi$. By a connection $\alpha$ form of $H$ we mean a mapping $\omega^{\alpha}: T^{\alpha} \Phi \rightarrow \mathfrak{g}^{\alpha}$ such that

1. $\omega^{\alpha} \mid \mathfrak{g}^{\alpha}=i d$,

2. $\operatorname{ker} \omega^{\alpha}=H$.

LEMMA 8.11. For the given connection $H$ its connection $\alpha$-form has the property: $\left(D_{h}\right)_{* g} \circ \omega_{1 g}^{\alpha}=\omega_{1 g h}^{\alpha} \circ\left(D_{h}\right)_{* g}$.

THEOREM 8.12. 1. If $H$ is a connection in the F-groupoid $\Phi$, then its connection $\alpha$-form $\omega^{\alpha}$ fulfils the condition:

(*) for any right-invariant vector field $X$ on $\Phi$ the mapping $\omega^{\alpha} \circ X$ is smooth.

2. If, for the F-groupoid $\Phi$ the sequence $A(\Phi) \underset{\text { Sec } \gamma}{\longrightarrow} \operatorname{Sec} E=X(E) \rightarrow 0$ is exact and the mapping $\omega^{\alpha}: T^{\alpha} \Phi \rightarrow \mathfrak{g}^{\alpha}$ is such that:

(a) $\omega^{\alpha} \mid \mathfrak{g}^{\alpha}=i d$,

(b) for any $X \in X^{R}(\Phi)$ the cross-section $\omega^{\alpha} \circ X$ is smooth,

(c) $\left(D_{h}\right)_{* g} \circ \omega_{1 g}^{\alpha}=\omega_{1 g h}^{\alpha} \circ\left(D_{h}\right)_{* g}$,

then ker $\omega^{\alpha}$ is some connection in the F-groupoid $\Phi$.

Proof. (1) Take any right-invariant vector field $X$ on $\Phi$. There exists a unique smooth cross-section $\xi \in \operatorname{Sec} A(\Phi)$ corresponding to it. The image $\gamma(\xi)$ of this cross-section by the anchor is a smooth cross-section of the semibundle $E$. Then its horizontal lifting to the smooth vector field $(\gamma(\xi))^{H} \in X(H)$ exists. Then $X=\left(X-(\gamma(\xi))^{H}\right)+(\gamma(\xi))^{H}$ is a smooth decomposition into the vertical part $X-(\gamma(\xi))^{H}$ and the horizontal part $(\gamma(\xi))^{H}$. So the mapping

$$
\omega^{\alpha} \circ X=\omega^{\alpha} \circ\left(X-(\gamma(\xi))^{H}\right)+\left((\gamma(\xi))^{H}\right)=X-(\gamma(\xi))^{H}
$$

is smooth.

(2) Suppose that $\omega^{\alpha}: T^{\alpha} \Phi \rightarrow g^{\alpha}$ is a mapping fulfilling conditions (a), (b), (c). The subspace $\operatorname{ker} \omega^{\alpha} \subset T^{\alpha} \Phi$ is a regular distribution on $\Phi$. It is sufficient to show that ker $\omega^{\alpha}$ fulfils conditions (1)-(3) from the definition of a connection 8.1. In fact, (1) follows from the diffeomorphy of $D_{h},(2)$ is obvious and condition (3) follows from the condition (a) of theorem 8.8 . 
Proposition 8.13. Let the mappings $k$ and $\bar{\alpha}$ be defined as follows

$$
\begin{aligned}
& k: \alpha^{*} \mathfrak{g} \rightarrow \mathfrak{g}^{\alpha}, \quad(h, v) \mapsto\left(A_{h}\right)_{* u_{\alpha h}}(v), \\
& \bar{\alpha}: \alpha^{*} \mathfrak{g} \rightarrow \mathfrak{g}^{\alpha}, \quad(h, v) \mapsto v .
\end{aligned}
$$

where $A_{h}: G_{\alpha h} \rightarrow \Phi_{\alpha h}, a \mapsto h \cdot a$. Then:

1. the mapping $k$ is an isomorphism on the fibres of vector semibundles over $\Phi$,

2. the diagram below commutes

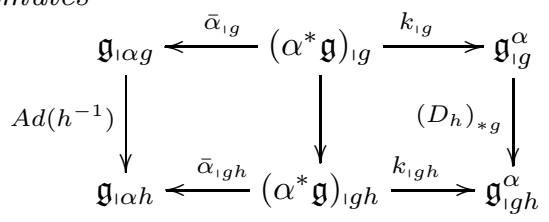

Let $\omega^{\alpha}: T^{\alpha} \Phi \rightarrow \mathfrak{g}^{\alpha}$ be an $\alpha$-form of some connection $H$ in the F-groupoid $\Phi$. Then the mapping $\bar{\omega}^{\alpha}:=\bar{\alpha} \circ k^{-1} \circ \omega^{\alpha}: T^{\alpha} \Phi \rightarrow \mathfrak{g}$ is a smooth $\alpha$-form of order 1 on $\Phi$ with values in the semibundle $\mathfrak{g}$.

Proposition 8.14. The form $\bar{\omega}^{\alpha}$ is Ad-equivariant.

Proof. Follows from the equality $\left(D_{h}\right)_{* g} \circ k_{ı g} \circ\left(\bar{\alpha}_{\imath g}\right)^{-1}=k_{ı g h} \circ\left(\bar{\alpha}_{i g h}\right)^{-1} \circ A d\left(h^{-1}\right)$.

Corollary 8.15. For any connection $\alpha$-form $\omega^{\alpha}$, we have $\bar{\omega}_{1 h}^{\alpha}=\operatorname{Ad}\left(h^{-1}\right) \circ \omega_{1 \beta h} \circ$ $\left(D_{h^{-1}}\right)_{*}$.

Definition 8.16. The form $\Psi \in \Omega^{\alpha}(\Phi ; \mathfrak{f})$ will be called a horizontal form if for any vertical vector field $X$ (i.e. for any cross-section of $\mathfrak{g}^{\alpha}$ ), $i_{X}^{\alpha, \mathfrak{f}} \Psi=0$.

Definition 8.17. The form $\Psi \in \Omega_{A}(M ; \mathfrak{f})$ will be called a horizontal form if $i_{\xi}^{A, \mathfrak{f}} \Psi=$ 0 for any $\xi \in S e c \mathfrak{g}$.

The horizontal forms form a vector space denoted by $\Omega_{A, i}(M ; \mathfrak{f})$. Moreover $\Omega_{A, i}(M)$ is an algebra and $\Omega_{A, i}(M ; \mathfrak{f})$ is a submodule of the $\Omega_{A, i}(M)$-module $\Omega_{A}(M ; \mathfrak{f})$.

Let $T$ be a fixed representation of the F-groupoid $\Phi$ in the vector semibundle $\mathfrak{f}$.

Definition 8.18. A form $\Psi \in \Omega^{\alpha}(\Phi ; \mathfrak{f})$ will be called a $T$-basic form (or, briefly, basic) if it is horizontal and $T$-equivariant.

The space of $T$-basic $\alpha$-forms will be denoted by $\Omega_{T, b}^{\alpha}(\Phi ; \mathfrak{f})$ (or $\Omega_{b}^{\alpha}(\Phi)$ if $T$ is trivial).

THEOREM 8.19. The space $\Omega_{b}^{\alpha}(\Phi)$ is an algebra. Moreover, the linear isomorphism $\tau_{T}$ restricts to a linear isomorphism $\tau_{T, i}: \Omega_{T, b}^{\alpha}(\Phi ; \mathfrak{f}) \rightarrow \Omega_{A, i}(M ; \mathfrak{f})$ and the mapping $\tau_{R, i}: \Omega_{b}^{\alpha}(\Phi) \rightarrow \Omega_{A, i}(M)$ is an isomorphism of algebras.

Let $\omega^{\alpha}: T^{\alpha} \Phi \rightarrow \mathfrak{g}^{\alpha}$ be a connection $\alpha$-form in the F-groupoid $\Phi$. Define

1. the horizontal projection of vectors $H^{\alpha}: T^{\alpha} \Phi \rightarrow T^{\alpha} \Phi$ by the formula $H^{\alpha}:=$ $i d-j^{\alpha} \circ \omega^{\alpha}$,

2. the horizontal projection of differential forms $H_{*}^{\alpha, \mathfrak{f}}: \Omega^{\alpha}(\Phi ; \mathfrak{f}) \rightarrow \Omega^{\alpha}(\Phi ; \mathfrak{f})$ by the formula $H_{*}^{\alpha, \mathfrak{f}} \Psi\left(h, v_{1}, \ldots, v_{q}\right)=\Psi\left(h ; H^{\alpha} v_{1}, \ldots, H^{\alpha} v_{q}\right)$,

3. the exterior covariant derivative in $\Phi$ with values in the semibundle $\mathfrak{f}$, associated with the connection form $\omega^{\alpha}$

$$
\nabla^{\alpha, \mathfrak{f}}: H_{*}^{\alpha, \mathfrak{f}} \circ d^{\alpha, \mathfrak{f}}
$$


THEOREM 8.20. 1. The forms $\Psi \in I m H_{*}^{\alpha, \mathfrak{f}}$ are horizontal.

2. The subspace $\Omega_{T}^{\alpha}(\Phi ; \mathfrak{f})$ of equivariant $\alpha$-forms with respect to a given representation $T$ is stable with respect to $H_{*}^{\alpha, \mathfrak{f}}$.

3. The operator $\nabla^{\alpha, \mathfrak{f}}$ carries equivariant forms over to T-basic forms.

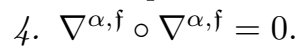

Definition 8.21. By the curvature $\alpha$-form of the connection $\alpha$-form $\omega^{\alpha}$ in the groupoid $\Phi$ we mean the $\alpha$-form $\Omega^{\alpha}:=\nabla^{\alpha, \mathfrak{g}} \omega^{\alpha}$ with values in $\mathfrak{g}$.

THEOREM 8.22. $\Omega^{\alpha}$ is a T-basic form.

Fix a connection $\lambda: E \rightarrow A(\Phi)$ in the F-algebroid $A(\Phi)=(A(\Phi), \llbracket \cdot, \cdot \rrbracket, \gamma)$ whose connection form is $\omega$ and take some vector semibundle $\mathfrak{f}$ over the manifold $M$ and a representation $T$ of the $\mathrm{F}$-groupoid $\Phi$ in $\mathfrak{f}$.

Definition 8.23. Let $\omega: A(\Phi) \rightarrow \mathfrak{g}$ be a connection form in the F-algebroid $A(\Phi)$. By the horizontal projection of vectors corresponding to the connection form $\omega$ we mean the mapping $H: A(\Phi) \rightarrow A(\Phi)$ defined by the formula $H:=i d-j \circ \omega$.

Definition 8.24. By the horizontal projection of forms in the F-algebroid $A(\Phi)$ we mean the linear mapping $H_{*}^{A, \mathfrak{f}}: \Omega_{A}(M ; \mathfrak{f}) \rightarrow \Omega_{A}(M ; \mathfrak{f})$ defined by the formula

$$
\left(H_{*}^{A, \mathfrak{f}} \Psi\right)\left(x ; v_{1}, \ldots, v_{q}\right)=\Psi\left(x ; H v_{1}, \ldots, H v_{q}\right),
$$

where $H: A(\Phi) \rightarrow A(\Phi)$ is a horizontal projection in the $\mathrm{F}$-algebroid $A(\Phi)$ corresponding to the connection form $\omega$. If $\mathfrak{f}=M \times \mathbb{R}$, then the letter $\mathfrak{f}$ will be omitted.

THEOREM 8.25. The operator $H_{*}^{A, \mathfrak{f}}$ has the following properties:

1. $H_{*}^{A, \mathfrak{f}} \mid \Omega_{A, i}(M ; \mathfrak{f})=i d$,

2. $\operatorname{Im} H_{*}^{A, \mathfrak{f}}=\Omega_{A, i}(M ; \mathfrak{f})$,

3. $H_{*}^{A, \mathfrak{f}}(\psi \wedge \Psi)=H_{*}^{A, \mathfrak{f}} \psi \wedge H_{*}^{A, \mathfrak{f}} \Psi$, for $\psi \in \Omega_{A}(M)$ and $\Psi \in \Omega_{A}(M ; \mathfrak{f})$,

4. the diagram below commutes

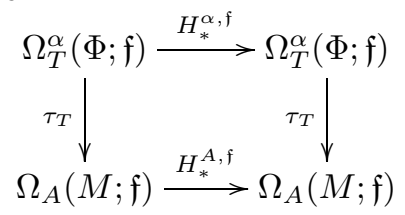

Definition 8.26. By the exterior covariant derivative in the F-algebroid $A(\Phi)$ with values in the semibundle $\mathfrak{f}$, associated with the connection $\lambda$ we shall mean the linear endomorphism $\nabla^{A, \mathfrak{f}}:=H_{*}^{A, \mathfrak{f}} \circ d^{A, \mathfrak{f}}$. If $\mathfrak{f}=M \times \mathbb{R}$, then the letter $\mathfrak{f}$ will be omitted.

THEOREM 8.27. The exterior covariant derivative $\nabla^{A, \mathfrak{f}}$ has the following properties:

1. $\operatorname{Im} \nabla^{A, \mathfrak{f}} \subset \Omega_{A, i}(M ; \mathfrak{f})$,

2. $\nabla^{A, \mathfrak{f}}(\psi \wedge \Psi)=\nabla^{A} \psi \wedge H_{*}^{A, \mathfrak{f}} \Psi+(-1)^{q} H_{*}^{A} \psi \wedge \nabla^{A, \mathfrak{f}} \Psi$, for $\psi \in \Omega_{A}^{q}(M)$ and $\Psi \in$ $\Omega_{A, i}(M ; \mathfrak{f})$,

3. $\nabla^{A, \mathfrak{f}} \circ \tau_{A d}=\tau_{A d} \circ \nabla^{A, \mathfrak{f}}$.

Our next step will be to define some endomorphism of the vector space $\Omega_{E}(M ; \mathfrak{f})$ of tangential forms with the help of an exterior covariant derivative in the algebroid $A(\Phi)$ 
with values in the semibundle $\mathfrak{f}$. For this purpose, define the mappings $\gamma_{\mathfrak{f}}^{*}: \Omega_{E}(M ; \mathfrak{f}) \rightarrow$ $\Omega_{A}(M ; \mathfrak{f})$ and $\lambda_{\mathfrak{f}}^{*}: \Omega_{A}(M ; \mathfrak{f}) \rightarrow \Omega_{E}(M ; \mathfrak{f})$ by the formulae:

$$
\begin{aligned}
\gamma_{\mathfrak{f}}^{*}(\Theta)\left(x ; v_{1}, \ldots, v_{q}\right) & =\Theta\left(x ; \gamma\left(v_{1}\right), \ldots, \gamma\left(v_{q}\right)\right), \\
\lambda_{\mathfrak{f}}^{*}(\Psi)\left(x ; w_{1}, \ldots, w_{q}\right) & =\Psi\left(x ; \lambda\left(w_{1}\right), \ldots, \lambda\left(w_{q}\right)\right)
\end{aligned}
$$

for $\Theta \in \Omega_{E}^{q}(M ; \mathfrak{f}), \Psi \in \Omega_{A}^{q}(M ; \mathfrak{f}), x \in M, v_{i} \in A_{1 x}, w_{i} \in E_{\mid x}, i=1, \ldots, q$.

Proposition 8.28. The mappings $\gamma_{\mathfrak{f}}^{*}$ and $\lambda_{\mathfrak{f}}^{*}$ are linear homomorphisms fulfilling the equalities: $\gamma_{\mathfrak{f}}^{*}(\theta \wedge \Theta)=\gamma_{\mathfrak{f}}^{*} \theta \wedge \gamma_{\mathfrak{f}}^{*} \Theta$ for $\theta \in \Omega_{E}(M), \Theta \in \Omega_{E}(M ; \mathfrak{f})$, and $\lambda_{\mathfrak{f}}^{*}(\psi \wedge \Psi)=$ $\lambda_{\mathfrak{f}}^{*} \psi \wedge \lambda_{\mathfrak{f}}^{*} \Psi$ for $\psi \in \Omega_{A}(M), \Psi \in \Omega_{A}(M ; \mathfrak{f})$.

COROLlary 8.29. If $\mathfrak{f}$ is a trivial bundle, the mappings $\gamma^{*}: \Omega_{E}(M) \rightarrow \Omega_{A}(M)$ and $\lambda^{*}: \Omega_{A}(M) \rightarrow \Omega_{E}(M)$ are algebra homomorphisms.

Proposition 8.30. 1. $\lambda_{\mathfrak{f}}^{*} \Theta \in \Omega_{A, i}(M ; \mathfrak{f})$ for $\Theta \in \Omega_{E}(M ; \mathfrak{f})$,

2. the mappings $\gamma_{\mathfrak{f}, i}^{*}: \Omega_{E}(M ; \mathfrak{f}) \rightarrow \Omega_{A, i}(M ; \mathfrak{f}), \Theta \mapsto \gamma_{\mathfrak{f}}^{*} \Theta$ and $\lambda_{\mathfrak{f}, i}^{*}: \Omega_{A, i}(M ; \mathfrak{f}) \rightarrow$ $\Omega_{E}(M ; \mathfrak{f}), \Psi \mapsto \lambda_{\mathfrak{f}}^{*} \Psi$ are linear isomorphisms. Moreover, the mappings $\gamma_{\mathfrak{i}}^{*}: \Omega_{E}(M)$ $\rightarrow \Omega_{A}(M)$ and $\lambda_{\mathfrak{i}}^{*}: \Omega_{A}(M) \rightarrow \Omega_{E}(M)$ are the isomorphisms of algebras.

Definition 8.31. The only endomorphism $\nabla^{\mathfrak{f}}$ of the vector space $\Omega_{E}(M ; \mathfrak{f})$ for which the diagram below commutes

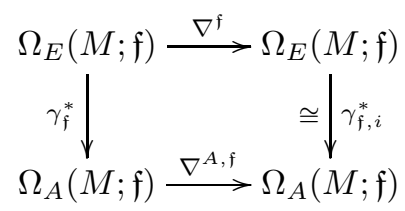

will be called the partial exterior covariant derivative in the semibundle $\mathfrak{f}$, associated with the connection $\lambda$.

Proposition 8.32. The endomorphism $\nabla^{\mathfrak{f}}$ has the following properties:

1. $\nabla^{\mathfrak{f}}=\lambda_{\mathfrak{f}, i}^{*} \circ \nabla^{A, \mathfrak{f}} \circ \gamma_{\mathfrak{f}}^{*}=\lambda_{\mathfrak{f}}^{*} \circ \nabla^{A, \mathfrak{f}} \circ \gamma_{\mathfrak{f}}^{*}$,

2. $\nabla^{\mathfrak{f}}=\lambda_{\mathfrak{f}}^{*} \circ d^{A, \mathfrak{f}} \circ \gamma_{\mathfrak{f}}^{*}$,

3. if $\mathfrak{f}=M \times \mathbb{R}$ is a trivial bundle, then $\nabla^{\mathfrak{f}}=d^{E}$, so $d^{E}=\lambda^{*} \circ d^{A} \circ \gamma^{*}$,

4. $\nabla^{\mathfrak{f}}(\theta \wedge \Theta)=d^{E} \theta \wedge \Theta+(-1)^{q} \theta \wedge \nabla^{\mathfrak{f}} \Theta$, for $\theta \in \Omega_{E}^{q}(M), \Theta \in \Omega_{E}(M ; \mathfrak{f})$,

5. $\left(\nabla^{\mathfrak{f} \Theta}\right)\left(X_{0}, \ldots, X_{q}\right)=\sum_{j=1}^{q}(-1)^{q} \nabla_{X_{j}}^{\mathfrak{f}}\left(\Theta\left(X_{0}, \ldots, \hat{X}_{j, \ldots, X_{q}}\right)\right)$

$+\sum_{i<j}(-1)^{i+j} \Theta\left(\left[X_{i}, X_{j}\right], \ldots, \hat{X}_{i}, \ldots, \hat{X}_{j}, \ldots, X_{q}\right)$

for $X_{0}, \ldots, X_{q} \in S e c E, \Theta \in \Omega_{E}^{q}(M ; \mathfrak{f})$.

Corollary 8.33. The endomorphism $\nabla^{\mathfrak{f}}$ restricted to Secf, i.e. $\nabla^{\mathfrak{f}}:$ Secf $\rightarrow$ $\Omega_{E}^{1}(M ; \mathfrak{f})$, is defined by the formula $\nabla_{X}^{\mathfrak{f}}(\sigma)=\left(T^{\prime} \circ \lambda \circ X\right)(\tilde{\sigma})$ for $\sigma \in \operatorname{Secf}, X \in$ SecE, and fulfils the conditions: (a) $\nabla^{\mathfrak{f}}$ is linear, (b) $\nabla_{f X}^{\mathfrak{f}} \sigma=f \cdot \nabla_{X}^{\mathfrak{f}} \sigma$, (c) $\nabla_{X}^{\mathfrak{f}} \sigma(f \cdot \sigma)=$ $X(f) \cdot \sigma+f \cdot \nabla_{X}^{\mathfrak{f}} \sigma$ for $f \in C^{\infty}(M), \sigma \in \operatorname{Secf}$.

COROLlary 8.34. The endomorphism $\nabla^{\mathfrak{f}}$ restricted to any leaf $L$ of the foliation $E$, i.e. the mapping $\nabla_{I_{L}}^{\mathfrak{f}}: \operatorname{Sec}\left(\mathfrak{f}_{1 L}\right) \rightarrow \Omega^{1}\left(L ; \mathfrak{f}_{1 L}\right)$, is the usual covariant derivative on the manifold $L$. 
Definition 8.35. The curvature form of the connection $\lambda$ is the form $\Omega:=\nabla^{A, \mathfrak{g}} \omega \in$ $\Omega_{A}^{2}(M ; \mathfrak{g})$, where $\omega$ is the connection form of $\lambda$.

Proposition 8.36. The connection form $\Omega$ has the following properties:

1. $\Omega \in \Omega_{A, i}^{2}(M ; \mathfrak{g})$, which implies, in particular, $H_{*}^{A, \mathfrak{g}} \Omega=\Omega$,

2. $\tau_{A d}\left(\Omega^{\alpha}\right)=\Omega$, where $\Omega^{\alpha}$ is the curvature $\alpha$-form of the connection form $\omega^{\alpha}$ in the F-groupoid $\Phi$.

Proof. (1) According to theorem 8.27, $\operatorname{Im} \nabla^{A, \mathfrak{f}} \subset \Omega_{A, i}(M ; \mathfrak{f})$ for any semibundle $\mathfrak{f}$. Additionally, $\Omega$ is a 2 -form with values in $\mathfrak{g}$, so $\Omega \in \Omega_{A, i}(M ; \mathfrak{g})$.

(2) The equalities $\tau_{T} \circ d^{A, \mathfrak{f}}=d^{\alpha, \mathfrak{f}} \circ \tau_{T}, \tau_{T} \circ H_{*}^{\alpha, \mathfrak{f}}=H_{*}^{A, \mathfrak{f}} \circ \tau_{T}$ and the definitions of the forms $\Omega$ and $\Omega^{\alpha}$ give: $\tau_{A d}\left(\Omega^{\alpha}\right)=\tau_{A d}\left(\nabla^{\alpha, \mathfrak{g}} \omega^{\alpha}\right)=\nabla^{A, \mathfrak{g}} \circ \tau_{A d} \omega^{\alpha}=\nabla^{A, \mathfrak{g}} \omega=\Omega$.

Corollary 8.37. $\Omega\left(\xi_{0}, \xi_{1}\right)=-\omega\left(\llbracket H \circ \xi_{0}, H \circ \xi_{1} \rrbracket\right)$ for $\xi_{0}, \xi_{1} \in \operatorname{Sec} A$.

Definition 8.38. The tangential curvature tensor of the connection $\lambda$ is the 2 -form

$$
\Omega_{b}:=\lambda_{\mathfrak{g}}^{*} \Omega \in \Omega_{E}^{2}(M ; \mathfrak{g})
$$

where $\Omega \in \Omega_{A}^{2}(M ; \mathfrak{g})$ is the curvature form of the connection $\lambda$.

Proposition 8.39. The tangential curvature tensor $\Omega_{b}$ has the following properties:

1. $\Omega_{b}\left(X_{1}, X_{2}\right)=-\omega\left(\llbracket \lambda \circ X_{1}, \lambda \circ X_{2} \rrbracket\right)$,

2. $\llbracket \lambda \circ X_{1}, \lambda \circ X_{2} \rrbracket=\lambda \circ\left[X_{1}, X_{2}\right]-\Omega_{b}\left(X_{1}, X_{2}\right)$,

3. $\Omega=0 \Longleftrightarrow \Omega_{b}=0$,

4. $\Omega_{b}=0 \Longleftrightarrow$ the Lie product of two horizontal vector fields is horizontal.

THEOREM 8.40. $\nabla^{\mathfrak{g}} \Omega_{b}=0$.

Proof. Follows from proposition 8.32, definition 8.38, the equality $\lambda \circ \gamma=H$, proposition $8.36(1)$ and theorem $8.27(3)$ and theorem $8.39(4)$.

9. The Chern-Weil homomorphism Let $\mathfrak{f}_{1}, \ldots, \mathfrak{f}_{k}, \mathfrak{f}$ be vector semibundles over the same differential manifold $M$ and let $\Gamma: \mathfrak{f}_{1} \times \ldots \times \mathfrak{f}_{k} \rightarrow \mathfrak{f}$ be a smooth $k$-linear homomorphism of semibundles.

1. For forms $\Psi_{i} \in \Omega^{\alpha, q_{i}}\left(\Phi, \mathfrak{f}_{i}\right), i=1, \ldots, k$, define the form $\Gamma_{*}^{\alpha}\left(\Psi_{1}, \ldots, \Psi_{k}\right) \in \Omega^{\alpha, q}(\Phi, \mathfrak{f})$, $q=\sum_{i=1}^{k} q_{i}$, by the formula:

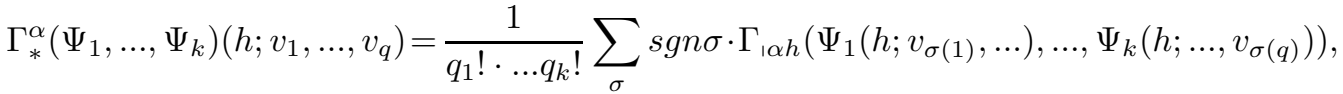
for $v_{i} \in\left(T^{\alpha} \Phi\right)_{\mid h}$. Note that $\Gamma_{*}^{\alpha}\left(\Psi_{1}, \ldots, \Psi_{k}\right)_{\mid x}=\left(\Gamma_{\mid x}\right)_{*}\left(\Psi_{1 \mid x}, \ldots, \Psi_{k \mid x}\right)$, where $\left(\Gamma_{\mid x}\right)_{*}$ is a standard operation on vector valued differential forms.

2. Similarly for forms $\Psi_{i} \in \Omega_{A}^{q_{i}}\left(M ; \mathfrak{f}_{i}\right), i=1, \ldots, k$, we define the form $\Gamma_{*}^{A}\left(\Psi_{1}, \ldots, \Psi_{k}\right)$ by the formula

$\Gamma_{*}^{A}\left(\Psi_{1}, \ldots, \Psi_{k}\right)\left(x ; v_{1}, \ldots, v_{q}\right)=\frac{1}{q_{1} ! \cdot \ldots q_{k} !} \sum_{\sigma} \operatorname{sgn} \sigma \cdot \Gamma_{\mid x}\left(\Psi_{1}\left(x ; v_{\sigma(1)}, \ldots\right), \ldots, \Psi_{k}\left(x ; \ldots, v_{\sigma(q)}\right)\right)$,

for $x \in M, v_{i} \in A_{1 x}$. 
In particular the form $\Gamma_{*}^{E}\left(\Theta_{1}, \ldots, \Theta_{k}\right) \in \Omega_{E}^{q}(M ; \mathfrak{f})$ is defined for $\Theta_{i} \in \Omega_{E}^{q_{i}}(M ; \mathfrak{f})$.

Let $\tau_{\mathfrak{f}}: \Omega^{\alpha}(\Phi ; \mathfrak{f}) \rightarrow \Omega_{A}(M ; \mathfrak{f})$ be the mapping given by $\tau_{\mathfrak{f}}(\Psi)(x)=\Psi\left(u_{x}\right), x \in M$. Note that $\tau_{\mathfrak{f}} \mid \Omega_{T}^{\alpha}(\Phi ; \mathfrak{f})=\tau_{T}$ for any representation $T$ of the F-groupoid $\Phi$ in the semibundle $\mathfrak{f}$. If $\mathfrak{f}=M \times \mathbb{R}$ then the letter $\mathfrak{f}$ will be omitted.

THEOREM 9.1. The homomorphisms $\Gamma_{*}^{A}$ and $\Gamma_{*}^{E}$ have the following properties:

1. $\Gamma_{*}^{A} \circ\left(\tau_{\mathfrak{f} 1} \times \ldots \times \tau_{\mathfrak{f} k}\right)\left(\Psi_{1}, \ldots, \Psi_{k}\right)=\tau_{\mathfrak{f}}\left(\Gamma_{*}^{\alpha}\left(\Psi_{1}, \ldots, \Psi_{k}\right)\right)$,

2. $\Gamma_{*}^{E} \circ\left(\lambda_{\mathfrak{f} 1} \times \ldots \times \lambda_{\mathfrak{f} k}\right)\left(\Psi_{1}, \ldots, \Psi_{k}\right)=\lambda_{\mathfrak{f}}\left(\Gamma_{*}^{A}\left(\Psi_{1}, \ldots, \Psi_{k}\right)\right)$,

3. $i_{X}^{\alpha, \mathfrak{f}}\left(\Gamma_{*}^{\alpha}\left(\Psi_{1}, \ldots, \Psi_{k}\right)\right)=\sum_{i=1}^{k}(-1)^{q_{1}+\ldots+q_{i-1}} \Gamma_{*}^{\alpha}\left(\Psi_{1}, \ldots, i_{X}^{\alpha, \mathfrak{f}_{i}} \Psi_{i}, \ldots, \Psi_{k}\right)$ for any $\alpha$-field $X$ and forms $\Psi_{i} \in \Omega^{\alpha}\left(\Phi ; \mathfrak{f}_{i}\right)$,

4. $i_{\xi}^{\alpha, \mathfrak{f}}\left(\Gamma_{*}^{\alpha}\left(\Psi_{1}, \ldots, \Psi_{k}\right)\right)=\sum_{i}(-1)^{q_{1}+\ldots+q_{i-1}} \Gamma_{*}^{\alpha}\left(\Psi_{1}, \ldots, i_{\xi}^{\alpha, \mathfrak{f}_{i}} \Psi_{i}, \ldots, \Psi_{k}\right)$ for any crosssection $\xi$ and forms $\Psi_{i} \in \Omega_{A}\left(M ; \mathfrak{f}_{i}\right)$,

5. $d^{\alpha, \mathfrak{f}}\left(\Gamma_{*}^{\alpha}\left(\Psi_{1}, \ldots, \Psi_{k}\right)\right)=\sum_{i}(-1)^{q_{1}+\ldots+q_{i-1}} \Gamma_{*}^{\alpha}\left(\Psi_{1}, \ldots, d^{\alpha, \mathfrak{f}_{i}} \Psi_{i}, \ldots, \Psi_{k}\right)$.

Definition 9.2. The k-linear homomorphism $\Gamma: \mathfrak{f}_{1} \times \ldots \times \mathfrak{f}_{k} \rightarrow \mathfrak{f}$ is called $\left(T_{1}, \ldots, T_{k}\right)$ invariant $\left(\left(T_{1}, \ldots, T_{k}\right)\right.$-invariant, if $T$ is the trivial representation $)$, if, for any $h \in \Phi$, the diagram below commutes $(x=\alpha h, y=\beta h)$ :

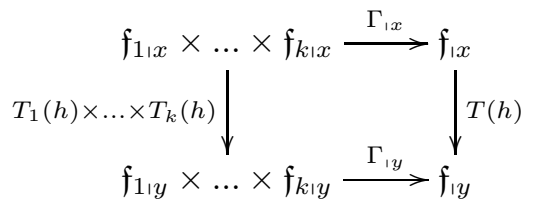

THEOREM 9.3. Let $\Gamma: \mathfrak{f}_{1} \times \ldots \times \mathfrak{f}_{k} \rightarrow \mathfrak{f}$ be a $\left(T_{1}, \ldots, T_{k} ; T\right)$-invariant homomorphism. Then

(a) $\Gamma_{*}^{\alpha}\left(\Psi_{1}, \ldots, \Psi_{k}\right) \in \Omega_{T}^{\alpha}(\Phi ; \mathfrak{f})$ for $\Psi_{i} \in \Omega_{T}^{\alpha}\left(\Phi ; \mathfrak{f}_{i}\right), i=1, \ldots, k$,

(b) $d^{A, \mathfrak{f}} \Gamma_{*}^{A}\left(\Psi_{1}, \ldots, \Psi_{k}\right)=\sum_{i=1}^{k}(-1)^{q_{1}+\ldots+q_{i-1}} \Gamma_{*}^{A}\left(\Psi_{1}, \ldots, d^{A, \mathfrak{f}} \Psi_{i}, \ldots, \Psi_{k}\right)$,

(c) $H_{*}^{A, \mathfrak{f}} \Gamma_{*}^{A}\left(\Psi_{1}, \ldots, \Psi_{k}\right)=\Gamma_{*}^{A}\left(H_{*}^{A, \mathfrak{f}_{1}} \Psi_{1}, \ldots, H_{*}^{A, \mathfrak{f}_{k}} \Psi_{k}\right)$ for $\Psi_{i} \in \Omega_{A}^{q_{i}}\left(M ; \mathfrak{f}_{i}\right)$,

(d) $\nabla^{\mathfrak{f}}\left(\Gamma_{*}^{E}\left(\Theta_{1}, \ldots, \Theta_{k}\right)\right)=\sum_{i=1}^{k}(-1)^{q_{1}+\ldots+q_{i-1}} \Gamma_{*}^{E}\left(\Theta_{1}, \ldots, \nabla^{\mathfrak{f}_{i}} \Theta_{i}, \ldots, \Theta_{k}\right)$, for $\Theta_{i} \in \Omega_{E}^{q_{i}}\left(M ; \mathfrak{f}_{i}\right)$.

In particular, for the trivial bundle $\mathfrak{f}=V \times \mathbb{R}$ we have:

(e) $d^{E}\left(\Gamma_{*}^{E}\left(\Theta_{1}, \ldots, \Theta_{k}\right)\right)=\sum_{i=1}^{k}(-1)^{q_{1}+\ldots+q_{i-1}} \Gamma_{*}^{E}\left(\Theta_{1}, \ldots, \nabla^{\mathfrak{f}_{i}} \Theta_{i}, \ldots, \Theta_{k}\right)$.

Let $\Phi$ be a fixed F-groupoid over the foliation $E$ and let 5.1 be its algebroid with the adjoint vector semibundle $\mathfrak{g}$. Let $\Gamma: \mathfrak{g} \times \ldots \times \mathfrak{g} \rightarrow \mathbb{R}$ be a $k$-linear homomorphism. Put

1. $\beta^{\alpha} \Gamma:=\Gamma_{*}^{\alpha}\left(\Omega^{\alpha}, \ldots, \Omega^{\alpha}\right) \in \Omega^{\alpha, 2 k}(\Phi)$,

2. $\beta^{A} \Gamma:=\Gamma_{*}^{A}(\Omega, \ldots, \Omega) \in \Omega_{A}^{2 k}(M)$,

3. $\beta^{E} \Gamma:=\Gamma_{*}^{E}\left(\Omega_{b}, \ldots, \Omega_{b}\right) \in \Omega_{E}^{\alpha, 2 k}(M)$

where $\Omega_{b}, \Omega, \Omega^{\alpha}$ are the curvature tensor of a given connection $\lambda$ in the algebroid $A$, the curvature form and the connection $\alpha$-form associated with the connection in the F-groupoid $\Phi$, respectively.

By theorem 9.1(3), theorem 9.3(a), theorem 8.25, theorem 9.1(4) and proposition 8.36 we have 
Proposition 9.4. If the homomorphism $\Gamma$ is $(A d, \ldots, A d)$-invariant, then

1. $\beta^{\alpha} \Gamma \in \Omega_{B}^{\alpha, 2 k}(\Phi)$,

2. $\beta^{A} \Gamma \in \Omega_{A, i}^{2 k}(M)$.

The space $\bigoplus^{k} \operatorname{Sec}\left(\mathfrak{L}^{k}(\mathfrak{g} ; \mathbb{R})\right)$ of all cross-sections of the set $\mathfrak{L}^{k}(\mathfrak{g} ; \mathbb{R})$ is supplied with the canonical structure $\left(\Gamma_{1}, \Gamma_{2}\right) \mapsto \Gamma_{1} \otimes \Gamma_{2}$ given by

$$
\left(\Gamma_{1} \otimes \Gamma_{2}\right)_{\mid x}=\Gamma_{1 \mid x} \otimes \Gamma_{2 \mid x},
$$

i.e. $\left(\Gamma_{1} \otimes \Gamma_{2}\right)_{\mid x}\left(v_{1}, \ldots, v_{k}\right)=\Gamma_{1 \mid x}\left(v_{1}, \ldots, v_{k}\right) \otimes \Gamma_{2 \mid x}\left(v_{1}, \ldots, v_{k}\right)$ for $\Gamma_{1} \in \mathfrak{L}^{k}(\mathfrak{g} ; \mathbb{R}), \Gamma_{2} \in$ $\mathfrak{L}^{l}(\mathfrak{g} ; \mathbb{R}), x \in M, v_{i} \in \mathfrak{g}_{1 x}$.

LEMMA 9.5. The set $\bigoplus^{k} \operatorname{Sec}\left(\mathfrak{L}^{k}(\mathfrak{g} ; \mathbb{R})\right)$ with the structure defined above forms an associative algebra with unity.

Proposition 9.6. The subspace $\bigoplus^{k} \operatorname{Sec}\left(\mathfrak{L}^{k}(\mathfrak{g} ; \mathbb{R})\right)_{I(\Phi)}$ of all $(A d, \ldots, A d)$-invariant cross-sections is a subalgebra of the algebra $\bigoplus^{k} \operatorname{Sec}\left(\mathfrak{L}^{k}(\mathfrak{g} ; \mathbb{R})\right)$.

TheOREM 9.7. The mapping $\beta^{\alpha}: \bigoplus^{k} \operatorname{Sec}\left(\mathfrak{L}^{k}(\mathfrak{g} ; \mathbb{R})\right) \rightarrow \Omega^{\alpha}(\Phi), \Gamma \mapsto \beta^{\alpha} \Gamma$, is an algebra homomorphism.

COROLlaRY 9.8. The mappings $\beta^{A}, \beta^{E}, \beta_{I}^{A}, \beta_{I}^{E}$ are homomorphisms of algebras.

Denote by $\mathfrak{L}_{s}^{k}(\mathfrak{g} ; \mathbb{R})$ the subspace of all symmetric $k$-linear homomorphisms and let $S: \mathfrak{L}^{k}(\mathfrak{g} ; \mathbb{R}) \rightarrow \mathfrak{L}_{s}^{k}(\mathfrak{g} ; \mathbb{R})$ be the symmetrization operator given by: $S_{1 x}(\Gamma)\left(v_{1}, \ldots, v_{k}\right)=$ $\frac{1}{k !} \sum_{\sigma} \Gamma\left(v_{\sigma(1)}, \ldots, v_{\sigma(k)}\right)$ for $x \in M, v_{i} \in \mathfrak{g}_{1 x}, \Gamma \in \mathfrak{L}^{k}(\mathfrak{g} ; \mathbb{R})_{\mid x}$.

The homomorphism $S \circ \Gamma$ is called a symmetric part of the homomorphism $\Gamma$ for $\Gamma \in \operatorname{Sec}\left(\mathfrak{L}^{k}(\mathfrak{g} ; \mathbb{R})\right)$.

Note that any $k$-linear homomorphism $\Gamma$ at any point $x \in M$ is a linear combination of homomorphisms of the form $\Gamma_{\mid x}=\Gamma_{1 \mid x} \otimes \ldots \otimes \Gamma_{k \mid x}$ for $\Gamma_{i} \in \operatorname{Sec}\left(\mathfrak{L}^{1}(\mathfrak{g} ; \mathbb{R})\right), i=1, \ldots, k$.

Proposition 9.9. The symmetric part of a homomorphism $\left(\Gamma_{1} \otimes \ldots \otimes \Gamma_{k}\right)_{\mid x}$ equals

$$
S \circ\left(\Gamma_{1} \otimes \ldots \otimes \Gamma_{k}\right)_{\mid x}=\frac{1}{k !} \sum_{\sigma}\left(\Gamma_{\sigma(1) ! x} \otimes \ldots \otimes \Gamma_{\sigma(k) \mid x}\right)
$$

for $x \in M, \Gamma_{i} \in \operatorname{Sec}\left(\mathfrak{L}^{1}(\mathfrak{g} ; \mathbb{R})\right), i=1, \ldots, k$.

Proposition 9.10. The value of $\beta^{\alpha}(\Gamma)$ depends only on the symmetric part of the homomorphism $\Gamma$.

Proposition 9.11. The symmetric part of the $(A d, \ldots, A d)$-invariant $k$-linear homomorphism is $(A d, \ldots, A d)$-invariant.

Proposition 9.11 implies that $S \circ \Gamma$ belongs to the domain of the homomorphism $\beta_{I}^{\alpha}$, if $\Gamma$ is an $(A d, \ldots, A d)$-invariant homomorphism. Then $\beta_{I}^{\alpha}(S \circ \Gamma)=\beta_{I}^{\alpha}(\Gamma)$. The space $\bigoplus^{k}\left(\operatorname{Sec}\left(\mathfrak{L}_{s}^{k}(\mathfrak{g} ; \mathbb{R})\right)\right)$ of symmetric homomorphisms possesses its own structure of an algebra with respect to the canonical symmetric product $\left(\Gamma_{1}, \Gamma_{2}\right) \mapsto \Gamma_{1} \vee \Gamma_{2}$ given by

$$
\left(\Gamma_{1} \vee \Gamma_{2}\right)_{\mid x}\left(v_{1}, \ldots, v_{k+l}\right)=\frac{1}{(k+l) !} \sum_{\sigma} \Gamma_{1 \mid x}\left(v_{\sigma(1)}, \ldots, v_{\sigma(k)}\right) \Gamma_{2 \mid x}\left(v_{\sigma(k+1)}, \ldots, v_{\sigma(k+l)}\right)
$$

for $\Gamma_{1} \in \operatorname{Sec}\left(\mathfrak{L}_{s}^{k}(\mathfrak{g} ; \mathbb{R})\right), \Gamma_{2} \in \operatorname{Sec}\left(\mathfrak{L}_{s}^{l}(\mathfrak{g} ; \mathbb{R})\right), x \in M$ and $v_{i} \in \mathfrak{g}_{1 x}$. Otherwise, $\Gamma_{1} \vee \Gamma_{2}=$ $S \circ\left(\Gamma_{1} \otimes \Gamma_{2}\right)$. 
LEMMA 9.12. The set $\bigoplus^{k}\left(\operatorname{Sec}\left(\mathfrak{L}_{s}^{k}(\mathfrak{g} ; \mathbb{R})\right)\right)$ with the above structure forms an associative algebra with unity.

Moreover, by propositions 9.9 and 9.11, the subspace $\bigoplus^{k}\left(\operatorname{Sec}\left(\mathfrak{L}_{s}^{k}(\mathfrak{g} ; \mathbb{R})\right)\right)_{I(\Phi)}$ of symmetric invariant homomorphisms is a subalgebra of the algebra $\bigoplus^{k}\left(\operatorname{Sec}\left(\mathfrak{L}_{s}^{k}(\mathfrak{g} ; \mathbb{R})\right)\right)$.

Now, define the mappings $\gamma^{\alpha}, \gamma^{A}, \gamma^{E}$ as restrictions of the mappings $\beta^{\alpha}, \beta^{A}, \beta^{E}$ to the algebra $\bigoplus^{k}\left(\operatorname{Sec}\left(\mathfrak{L}_{s}^{k}(\mathfrak{g} ; \mathbb{R})\right)\right)$ and the mappings $\gamma_{I}^{\alpha}, \gamma_{I}^{A}, \gamma_{I}^{E}$ as restrictions of the mappings $\beta^{\alpha}, \beta^{A}, \beta^{E}$ to the algebra $\bigoplus^{k}\left(\operatorname{Sec}\left(\mathfrak{L}_{s}^{k}(\mathfrak{g} ; \mathbb{R})\right)\right)_{I(\Phi)}$, respectively.

Proposition 9.13. The mapping $\gamma^{\alpha}$ is a homomorphism of algebras.

Corollary 9.14. The mappings $\gamma^{A}, \gamma^{E}$ and $\gamma_{I}^{\alpha}, \gamma_{I}^{A}, \gamma_{I}^{E}$ are homomorphisms of algebras.

THEOREM 9.15. $d^{E} \circ \gamma_{I}^{E}=0$.

Theorem 9.15 implies that $\operatorname{Im} \gamma_{I}^{E} \subset Z(E) \subset \Omega_{E}(M)$.

Definition 9.16. The Chern-Weil homomorphism of the F-groupoid $\Phi$ is the superposition

$$
h_{\Phi}: \bigoplus^{k}\left(\operatorname{Sec}\left(\mathfrak{L}_{s}^{k}(\mathfrak{g} ; \mathbb{R})\right)\right)_{I(\Phi)} \rightarrow Z(E) \rightarrow H_{E}(M), \quad \Gamma \mapsto\left[\gamma_{I}^{E}(\Gamma)\right] .
$$

The image of the mapping $h_{\alpha}$ is a graded subalgebra of the tangential cohomology algebra $H_{E}(M)$ called the Pontryagin algebra [K3] of the F-groupoid $\Phi$ and denoted by Pont $\Phi$. The main property of the mapping $h_{\Phi}$ is described by the following

TheOREM 9.17. The Chern-Weil homomorphism $h_{\Phi}$ is independent of the choice of a connection.

Let us make a few assumptions common to the three lemmas below. Let $\Phi$ and $\Phi^{\prime}$ be any two F-groupoids over the foliations $E$ and $E^{\prime}$ of the manifolds $M$ and $M^{\prime}$, respectively. Let $(A, \llbracket \cdot, \cdot \rrbracket, \gamma)$ and $\left(A^{\prime}, \llbracket \cdot, \cdot \rrbracket^{\prime}, \gamma^{\prime}\right)$ be their F-algebroids. Let $F: \Phi \rightarrow \Phi^{\prime}$ be a smooth homomorphism of F-groupoids over the mapping $f: M \rightarrow M^{\prime}$, and let $\omega: A \rightarrow \mathfrak{g}$ and $\omega^{\prime}: A^{\prime} \rightarrow \mathfrak{g}^{\prime}$ be any connection forms of $A$ and $A^{\prime}$, respectively, such that the diagram below commutes

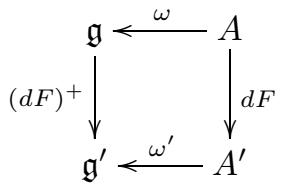

The mappings $d F$ and $(d F)^{+}$are the appropriate restrictions of the mapping $F_{*}: T \Phi \rightarrow$ $T \Phi^{\prime}$.

Lemma 9.18. For any $x \in M$ the diagram

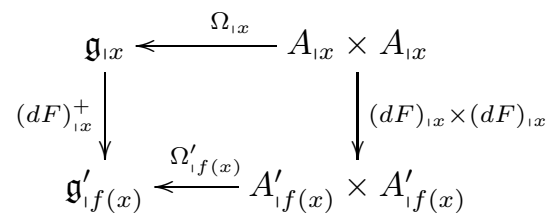

commutes. 
LEMMA 9.19. If $\Omega_{b}$ and $\Omega_{b}^{\prime}$ are the curvature tensors of the curvature forms $\Omega$ and $\Omega^{\prime}$, then the diagram

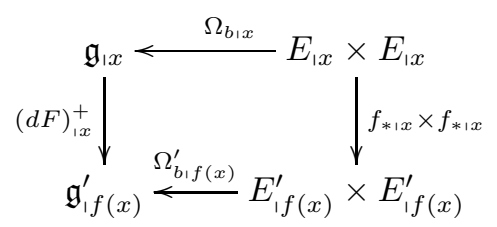

commutes $\left(E=T E, E^{\prime}=T E^{\prime}\right)$.

Lemma 9.20. The Chern-Weil homomorphisms $h_{\Phi}$ and $h_{\Phi^{\prime}}$ built with the use of the forms $\omega$ and $\omega^{\prime}$ give a commutative diagram

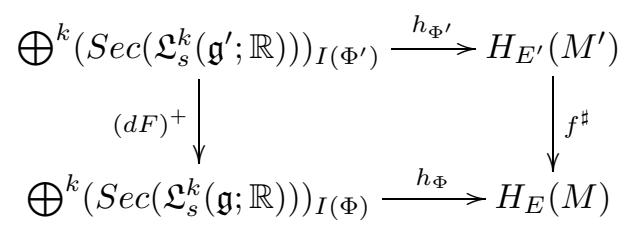

where $E=T E, E^{\prime}=T E^{\prime}$ and, for $\Gamma^{\prime} \in \operatorname{Sec}\left(\mathfrak{L}_{s}^{k}\left(\mathfrak{g}^{\prime} ; \mathbb{R}\right)\right)$, the mapping $(d F)^{+*}\left(\Gamma^{\prime}\right)$ is the $k$-linear homomorphism $\mathfrak{g} \times \ldots \times \mathfrak{g} \rightarrow \mathbb{R}$ given by

$$
(d F)^{+*}\left(\Gamma^{\prime}\right)\left(x ; v_{1}, \ldots, v_{k}\right)=\Gamma^{\prime}\left(f(x) ;(d F)_{1 x}^{+}\left(v_{1}\right), \ldots,(d F)_{1 x}^{+}\left(v_{k}\right)\right) .
$$

Proof of theorem 17. Consider the Cartesian product $\Phi \times \mathbb{R}^{2}$ of the F-groupoid $\Phi$ and the trivial groupoid $\mathbb{R}^{2}$. Theorem 5.5 implies that it is an F-groupoid over the foliation $E \times \mathbb{R}=\{L \times \mathbb{R} ; L \in E\}$ of the manifold $M \times \mathbb{R}$. In this groupoid the source and target mappings $\tilde{\alpha}$ and $\tilde{\beta}$ are defined by $\tilde{\alpha}(h, x, y)=(\alpha h, y)$ and $\tilde{\beta}(h, x, y)=(\beta h, y)$. The leaf of the F-groupoid $\Phi \times \mathbb{R}^{2}$ over the point $(x, t) \in M \times \mathbb{R}$ is $\Phi_{x} \times(\{t\} \times \mathbb{R})$. The algebroid of the F-groupoid $\Phi \times \mathbb{R}^{2}$ is the Cartesian product $A \times T \mathbb{R}$ defined in theorem 6.1. The Atiyah sequence of this algebroid has the form $0 \rightarrow \mathfrak{g} \times 0 \hookrightarrow A \times T \mathbb{R} \stackrel{\gamma \times i d}{\longrightarrow} E \times T \mathbb{R} \rightarrow 0$. Consider the projection $\Phi \times \mathbb{R}^{2} \rightarrow \Phi$. It is a homomorphism of F-groupoids which defines a homomorphism of vector semibundles, $j=0,1$,

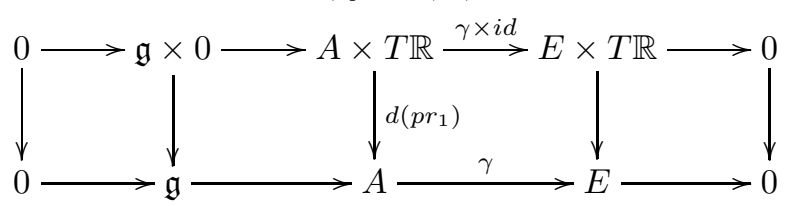

Now, let $\omega_{0}: A \rightarrow \mathfrak{g}$ and $\omega_{1}: A \rightarrow \mathfrak{g}$ be any two connection forms in the algebroid $A$. Put $\tilde{\omega}_{\mid(x, t)}(v, w):=\left(\omega_{0 \nmid x}(v) \cdot(1-t)+\omega_{1 \mid x}(v) \cdot y, 0\right)$. This formula defines some connection form $\tilde{\omega}: A \times T \mathbb{R} \rightarrow \mathfrak{g} \times\{0\}$ in the algebroid $A \times T \mathbb{R}$.

Consider the following homomorphisms of F-groupoids $F_{1}: \Phi \rightarrow \Phi \times \mathbb{R}^{2}, h \mapsto(h, 0,0)$, $F_{2}: \Phi \rightarrow \Phi \times \mathbb{R}^{2}, h \mapsto(h, 1,1)$ over the mappings $i_{0}: M \rightarrow M \times \mathbb{R}, x \mapsto(x, 0)$, $i_{1}: M \rightarrow M \times \mathbb{R}, x \mapsto(x, 1)$, respectively. Then we have the commutative diagrams

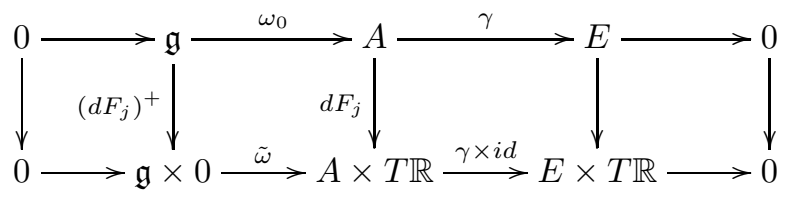


They allow one to use the previous lemma to get the commutativity of the diagrams, $j=0,1$,

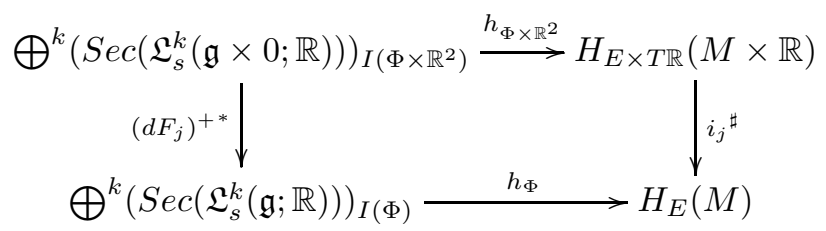

In the next step we shall prove the equality $i_{0}^{\sharp}=i_{1}^{\sharp}$. It follows from

LEMMA 9.21. Let $M$ and $N$ be two manifolds over the foliations with singularities $E$ and $E^{\prime}$, respectively. If $f, g: M \rightarrow N$ are smooth mappings and $H: M \times \mathbb{R} \rightarrow N$ is a homotopy between them such that, for any leaf $L \in E$ the image $H(L \times \mathbb{R})$ is contained in some leaf of the foliation $E^{\prime}$, then

$$
f^{\sharp}=g^{\sharp}: H_{E}(M) \rightarrow H_{E^{\prime}}(N),
$$

where $E=T E, E^{\prime}=T E^{\prime}$.

Coming back to the proof of the theorem, take the homotopy $H: i d_{M \times \mathbb{R}}: M \times \mathbb{R} \rightarrow$ $M \times \mathbb{R}$ joining the mappings $i_{0}$ and $i_{1}$. According to lemma 9.21 we have $i_{0}^{\sharp}=i_{1}^{\sharp}$. Now, consider the homomorphism $p_{1}: \mathfrak{g} \times\{0\} \rightarrow \mathfrak{g},(v, 0) \mapsto v$. Then $p_{1} \circ\left(d F_{0}\right)^{+}=i d_{\mathfrak{g}}$ and $p_{1} \circ\left(d F_{1}\right)^{+}=i d_{\mathfrak{g}}$. So

$$
\mathrm{id}: \operatorname{Sec}\left(\mathfrak{L}_{s}^{k}(\mathfrak{g} ; \mathbb{R})\right) \rightarrow \operatorname{Sec}\left(\mathfrak{L}_{s}^{k}(\mathfrak{g} \times 0 ; \mathbb{R})\right) \rightarrow \operatorname{Sec}\left(\mathfrak{L}_{s}^{k}(\mathfrak{g} ; \mathbb{R})\right) .
$$

Now, we can consider the commutative diagram

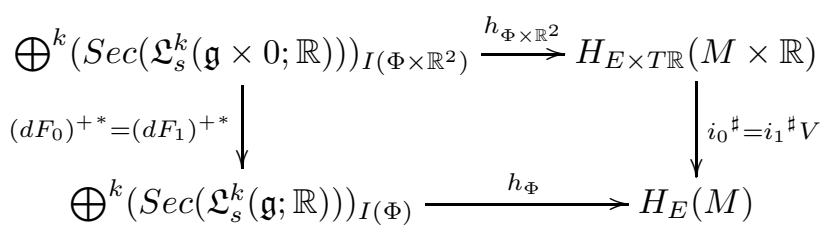

which implies

$$
h_{\Phi}=h_{\Phi} \circ\left(d F_{0}\right)^{+*} \circ p_{1}^{*}=i_{0}^{\sharp} \circ h_{\Phi \times \mathbb{R}^{2}} \circ p_{1}^{*}
$$

and

$$
h_{\Phi}=h_{\Phi} \circ\left(d F_{1}\right)^{+*} \circ p_{1}^{*}=i_{1}^{\sharp} \circ h_{\Phi \times \mathbb{R}^{2}} \circ p_{1}^{*} .
$$

Then the Chern-Weil homomorphism $h_{\Phi}$ is independent of the choice of a connection.

EXAMPLE 2. If $\Phi$ is a Pradines type groupoid over the foliation without singularities the Chern-Weil homomorphism is the same as the one defined in [K5].

ExAmple 3 . Let $\Psi=(\Psi, \alpha, \beta, M, \cdot)$ be a Lie groupoid and let $\Phi=(\alpha, \beta)^{-1}[R]$ be a groupoid of the equivalence relation $R$ determined by the Stefan foliation with singularities of the manifold $M$. Then the elements of the form $\sum_{i} f_{i} \Gamma_{i}$, where $f_{i}$ are the foliated functions and $\Gamma_{i}$ are $A d$-invariant cross-sections of the groupoid $\Psi$, are contained in the domain of the homomorphism $h_{\Phi}$ (see also [K7]). 


\section{References}

[H] J. Huebschmann, Extension of Lie-Rinehart algebras and the Chern-Weil construction, Contemp. Math. 227, 1999.

[K1] J. Kubarski, Smooth groupoids over foliations and their algebroids. Part I, Preprint No 1, Instytut Matematyki Politechniki Łódzkiej, 1986.

[K2] J. Kubarski, Pradines type groupoids, in: Proc. Winter School Geometry and Physics, Srni, January 1987, Czechoslovakia.

[K3] J. Kubarski, Pontryagin algebra of a transitive Lie algebroid, in: Proc. Winter School Geometry and Physics, Srni, January 1989, Czechoslovakia.

[K4] J. Kubarski, About Stefan's definition of a foliation with singularities: a reduction of the axioms, Bull. Soc. Math. France 118, (1990), 391-394.

[K5] J. Kubarski, Pradines-type groupoids over foliations; cohomology, connections and the Chern-Weil homomorphism, Preprint No 2, Instytut Matematyki Politechniki Łódzkiej, 1986.

[K6] J. The Chern-Weil homomorphism of regular Lie algebroids Kubarski, Publ. Dep. Math. Université de Lyon 1,

[K7] J. Kubarski, Characteristic classes of some Pradines-type groupoids and a generalization of the Bott vanishing theorem, in: Diff. Geom. and its Appl., Proc. Conf., August 24-30, 1986, Brno, Czechoslovakia.

[L1] K. Lisiecki, Foliated groupoids, Proc. Winter School Geometry and Physics, Srni, January 1989, Czechoslovakia.

[L2] K. Lisiecki, Koneksje w grupoidach sfoliowanych, Ph. D. Thesis, 1996, in Polish.

[SA] W. Sasin, Infinite cartesian product of differential groups, Math. Nachr. 149 (1990), $61-70$.

[SI] R. Sikorski, Abstract covariant derivative, Coll. Math. 18 (1967), 251-272.

[ST1] P. Stefan, Accessibility and foliations with singularities, Bull. Amer. Math. Soc. 80 (1974), 1142-1145.

[ST2] P. Stefan, Accessible sets, orbits, and foliations with singularities, Proc. London Math. Soc. 29 (1974), 699-713. 